\title{
Small resolutions of SU(5)-models
}

\section{in F-theory}

\author{
Mboyo Esole ${ }^{1,2}$ and Shing-Tung Yau ${ }^{1}$ \\ ${ }^{1}$ Department of Mathematics, Harvard University, Cambridge, \\ MA 02138, USA \\ esole@math.harvard.edu \\ ${ }^{2}$ Jefferson Physical Laboratory, Harvard University, Cambridge, \\ MA 02138, USA \\ yaumath.harvard.edu
}

\begin{abstract}
We provide an explicit desingularization and study the resulting fiber geometry of elliptically fibered four-folds defined by Weierstrass models admitting a split $\tilde{A}_{4}$ singularity over a divisor of the discriminant locus. Such varieties are used to geometrically engineer SU(5) grand unified theories in F-theory. The desingularization is given by a small resolution of singularities. The $\tilde{A}_{4}$ fiber naturally appears after resolving the singularities in codimension-one in the base. The remaining higher codimension singularities are then beautifully described by a four-dimensional affine binomial variety which leads to six different small resolutions of the elliptically fibered four-fold. These six small resolutions define distinct four-folds connected to each other by a network of flop transitions forming a dihedral group. The location of these exotic fibers in the base is mapped to conifold points of the three-folds that defines the type IIB orientifold limit of the F-theory. The full resolution has interesting properties, specially for fibers in codimension-three: the rank of the singular
\end{abstract}

e-print archive: http://lanl.arXiv.org/abs/1107.0733v2 
fiber does not necessary increase and the fibers are not necessary in the list of Kodaira and some are not even (extended) Dynkin diagrams.

\section{Contents}

1 Introduction

1.1 Fiber degenerations and F-theory

1.2 SU(5) unification in F-theory

1202

1.3 The conjectured fiber geometry of SU(5) models

1.4 Mathematical interests of the SU(5) model

1.5 Summary of results

1.6 Structure of the paper

1213

2 Elliptic fibrations and collisions of singularities

2.1 Elliptic fibrations and Weierstrass models

2.2 Kodaira classification and Tate algorithm

2.3 Miranda models and collisions of singular fibers

2.4 Szydlo generalization of Miranda models

2.5 F-theory versus Miranda models

3 Geometric engineering of SU(5) models in F-theory

3.1 Conjectured fiber geometry

4 Resolution over codimension-one loci in the base

4.2 Fiber mutation over higher codimension singularities 
5 The binomial geometry of SU(5) GUTs

5.1 Atiyah's flop and the small resolution of the conifold

5.2 Toric description of the binomial variety

5.3 Small resolutions and network of flop transitions

5.4 Algebraic description of the network of small resolutions

6 Small resolutions for SU(5) GUTs

6.1 Codimension-two singular fibers: $\tilde{A}_{5}$ and $\tilde{D}_{5}$ enhancements

6.2 An affine $\tilde{D}_{6}$ enhancement in codimension-three

6.3 Exotic fibers in codimension-three

6.4 Flop transitions and codimension-three singular fibers

6.5 Some comments on the exotic fibers

7 Conclusions and discussions

\section{Introduction}

The theory of elliptic curves is an elegant and vast subject in mathematics that can be traced back to ancient Greece and beyond. An elliptic curve is a non-singular irreducible curve of genus one with a distinguished point on it. It is well known that elliptic curves play an important role in number theory and are instrumental in cryptography and geometric modeling. Elliptic curves have also invaded many branches of theoretical physics through their modular properties. They are also familiar in several string theory dualities. 
The ultimate example of applications of elliptic curves to string theory is probably F-theory.

F-theory was introduced by Vafa [5] as a non-perturbative formulation of type IIB string theory providing a geometrization of its non-perturbative $\mathrm{SL}(2, \mathbb{Z})$ symmetry known as $S$-duality. F-theory provides a geometric formulation of type IIB string theory compactification on a complex variety $B$ with a varying axio-dilaton field $\tau$. The variation of the axio-dilaton field is geometrically realized in F-theory by an elliptic fibration $\pi: Y \rightarrow B$ where the variety $B$ is the base of the fibration:

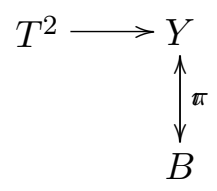

The period modulus of the elliptic fiber is interpreted as the axio-dilaton field:

$$
T^{2} \simeq \mathbb{C} /(\mathbb{Z}+\tau \mathbb{Z}), \quad \tau=C_{0}+\mathrm{ie}^{-\phi}, \quad \mathrm{e}^{\phi}=g_{s} .
$$

The S-duality group is then geometrically realized as the $\mathrm{SL}(2, \mathbb{Z})$ modular group of the elliptic fiber. The axio-dilaton field can be used to probe the presence of $(p, q)$-seven-branes since seven-branes and D-instantons are magnetic duals. In F-theory, the elliptic fibration is a Calabi-Yau space and the base of the fibration is usually a complex curve, surface or three-fold leading, respectively (after compactification) to a theory in 8,6 or 4 real spacetime dimensions [5-7]. There is another road to F-theory by starting from M-theory, a theory defined in a 11-dimensional spacetime. The duality between F-theory and M-theory is understood via a chain of dualities. Type IIB string theory compactified on a circle is T-dual to type IIA on a dual circle, which is then dual to M-theory compactified on a two-torus [8]:

$$
\text { M-theory } \stackrel{\text { S-duality }}{\longleftrightarrow} \text { IIA } \stackrel{\text { T-duality }}{\longleftrightarrow} \text { IIB } \longleftrightarrow \text { F-theory }
$$

In F-theory, one usually starts with a singular elliptic fibration given by a singular Weierstrass model and the smooth elliptic fibration is obtained by a resolution of the singularities of the elliptic fibration. Starting from a smooth elliptic fibration $Y$, the type IIB limit of F-theory is taken in two steps: first, shrink to zero area all fiber components not meeting the chosen section, then shrink the remaining components of each fiber to zero area. For singular fibers over codimension-one loci in the base, the structure of the singular fibers can be deduced without performing an explicit resolution thanks to Tate's algorithm. But this is not true for singular fibers that 
project to codimension-two or higher in the base. A resolution of singularity only changes the singular part of the variety while it is an isomorphism on the smooth part. Even so, a resolution is in general not unique and the singular part of the variety is usually replaced by a locus of higher dimension, typically a divisor. Additional requirements can be imposed on a resolution. One can, for example, ask the resolution to be small so that a singular locus of codimension $k$ is replaced by a locus which is smaller than a divisor. A more restricted definition of small resolution requires that a singular locus of codimension $k$ is replaced by a locus of codimension less than $k / 2$. Small resolutions are not always possible. One can also ask the resolution to be crepant. This is very common in string theory as it ensures that the Calabi-Yau condition is preserved. In this paper, we will analyze a particular model where F-theory is defined from a singular geometry for which the desingularization has been conjectured to have a remarkable structure but has never been analyzed systematically. We will use this specific example as an appetizer to attack a more general questions also relevant for mathematics.

We will consider an elliptic fibration endowed with a smooth section $\iota: B \rightarrow Y$. Fiberwise, the section selects a point on every fiber so that the fiber is a bona fide elliptic curve. In F-theory, the section provides a concrete embedding of the base $B$ (on which type IIB is compactified) inside the elliptic fibration $Y$. An elliptic fibration with a section has the nice property of being birationally equivalent to a (singular) Weierstrass model. Our model of interest will be given explicitly by a Weierstrass model in Tate's form

$$
\mathscr{E}: z y^{2}+a_{1} x y z+a_{3} y z^{2}=x^{3}+a_{2} x^{2} z+a_{4} x z^{2}+a_{6} z^{3},
$$

where $[x: y: z]$ are projective coordinates of a $\mathbb{P}^{2}$ bundle over the base $B$ and the coefficients $a_{i}$ are sections of appropriate line bundles. We will come back to the definition in Section 2. In this paper, the base of the fibration is a complex variety $B$ of complex dimension three (a three-fold) so that after the compactification we end up with a theory in four spacetime dimensions.

The elliptic fibration we will be interested in, is given by a singular Weierstrass model with a specific type of singularities (a $\tilde{A}_{4}$ singularity) over a divisor of the base. This singularity is motivated by the geometric engineering of $\mathrm{SU}(5)$ grand unified theories in F-theory. We will resolve the singularities and study carefully the resulting fiber geometry. Over the divisor over which the singularity is defined, we will get a singular fiber of type $I_{5}$ in Kodaira's notation and the singular fiber will degenerate further over certain curves and points in the base. More precisely, the fiber is a split $I_{5}$, meaning that each components of the singular fiber is defined without requiring a field extension. We assume that after the resolution, we have a smooth 
elliptic fibration with a smooth section and the fibration is required to be flat (all fibers have the same dimension) and the resolution to be crepant. All the results will be independent of the Calabi-Yau condition. The structure of the resolved geometry is conjectured in the physics literature to naturally realize a cascade of specializations to other types of singular fibers over certain loci of the base in order to geometrically engineer the matter content and the Yukawa couplings of the SU(5) grand unified theory.

In this paper, our point of view will be to use the physics as a motivation for the geometry we are studying. But all the results of this paper rely solely on a mathematical analysis. We will be able not only to resolve the geometry but we also present certain transitions between different resolutions of the singularities not anticipated by physicists. In the rest of the introduction, we will discuss the fiber geometry in F-theory, present the problem that we want to attack and its physical origin and summarize our results. The rest of the paper will be a systematic derivation of these results.

\subsection{Fiber degenerations and F-theory}

As it is usually the case for fibrations, many interesting properties of an elliptic fibration are encoded in the structure of its singular fibers. In F-theory, the structure of the singular fibers is a central piece in the dictionary between geometry and physics. In the presence of a $(p, q)$-seven-brane, the axio-dilaton field undergoes $\mathrm{SL}(2, \mathbb{Z})$-monodromies that determine the type of $(p, q)$-seven-branes. From the point of view of the elliptic fibration, such monodromies require the elliptic fiber to be singular over certain divisors of the base $B$. It follows that singular fibers geometrically detect the locations (in the base $B$ ) and types of $(p, q)$ seven-branes. The locus of points of the base over which the elliptic fiber is singular is called the discriminant locus. For an elliptic fibration given by a Weierstrass model, the discriminant locus is a divisor in the base $B$ defined by the equation $\Delta=0$, where $\Delta$ is defined in equation (2.9) or equivalently in equation (2.11). The discriminant locus can be composed of several irreducible components of different multiplicities $\Delta=\prod_{i} \Delta_{i}^{m_{i}}$ and can admit a sophisticated structure of singularities. The generic fiber over a component of the discriminant locus can degenerate further at the intersection with another component or more generally at a singularity of the discriminant locus. Such degenerations occur in codimension-two or higher in the base and are called collisions of singular fibers. Here, we will restrict ourself to flat fibrations so that all the fibers have the same dimension. In particular, a singular fiber is composed of a finite number of intersecting irreducible curves. The singular fibers of a smooth Weierstrass model are nodal and cuspidal irreducible curves. When 


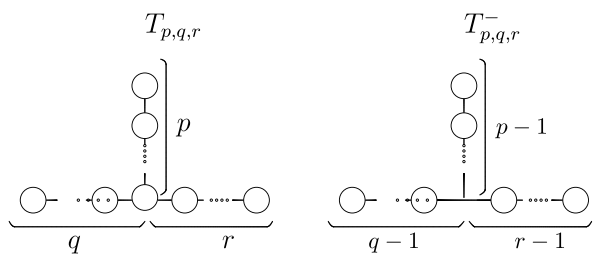

Figure 1: Fiber of type $T_{p, q, r}$ and $T_{p, q, r}^{-}$with $1 \leq p \leq q \leq r$. The fiber of type $T_{p, q, r}^{-}$is obtained from $T_{p, q, r}$ by replacing the central node by a point common to the three branches. A fiber of type $T_{p, q, r}$ has rank $p+q+r-2$, while a fiber of type $T_{p, q, r}^{-}$has rank $p+q+r-3$.

the Weierstrass model is singular one can get in addition a rich spectrum of reducible singular fibers after resolving the singularities. To each reducible singular fiber, it is convenient to associate a dual graph representing the intersection structure of the irreducible rational curves composing the singular fiber. The dual graph can take several shapes. It is a beautiful aspect of the theory of elliptic fibrations that the dual graph of the singular fiber over a generic point of a component of the discriminant locus is an ADE Dynkin diagram plus an extra node. We will denote projective ADE Dynkin diagrams by the corresponding Lie algebra notation $A_{n}, D_{n}$ and $E_{n}$ where the index $n$ refers to the number of nodes of the diagram and therefore to the rank of the Lie algebra. The associated affine ADE Dynkin diagrams will be denoted with a tilde: $\tilde{A}_{n}, \tilde{D}_{n}, \tilde{E}_{n}$. In the case of affine Dynkin diagrams, the number of nodes is $(n+1)$. To cover all the type of diagrams that will occur in this paper, it is useful to introduce the graphs of type $T_{p, q, r}$ and $T_{p, q, r}^{-}$. They are presented in figure 1 . They generalize diagrams of type $\tilde{E}_{n}$, $E_{n}, D_{n}$ and $A_{n}$.

The common wisdom on the physical meaning of singular fibers in F-theory can be summarized in three points:

(1) Each irreducible component of the discriminant locus corresponds to a divisor wrapped by $(p, q)$-seven-branes. The gauge group associated to branes wrapping a divisor of the discriminant locus is determined by the type of singular fibers above that component. In the spirit of Mckay's correspondence, the type of singular fibers over a component of the discriminant locus identifies the non-Abelian gauge group living on the brane wrapping that component ${ }^{1}$.

\footnotetext{
${ }^{1}$ If the fiber is not split, there is monodromy that exchange certain components of the singular fiber and by identifying such components, we can also get non-simply laced gauge groups.
} 
Table 1: F-theory description of gauge theories.

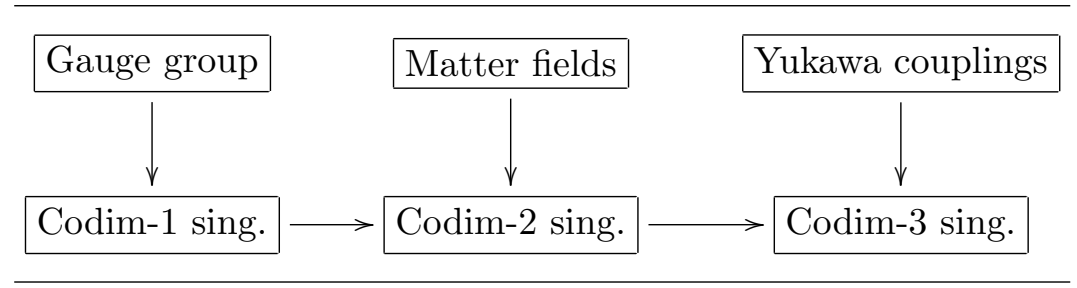

(2) Singular fibers in codimension-two in the base are related to charged chiral matter following Katz-Vafa's approach and the matter content can be read off following the branching rules of representation theory $[9,11,12]$. When the base is a three-fold, these codimensiontwo loci are called matter curves. This follows from a familiar picture in D-brane modeling under which chiral matter can be localized at D-branes intersections. In F-theory, this point has been supported by direct comparisons with the heterotic string in the case of elliptic three-folds leading to theories in six spacetime dimensions.

(3) Codimension-three singularities are related to Yukawa couplings (table 1).

When the singularities are more involved, already the second step (identification of the matter representation) can become delicate to handle [36]. Another approach based on the field theory on the seven-branes world volume has been developed recently [23-25,30]. However, the relation between the details of the field theory on the world volume of the seven branes and the singular fibers of the elliptic fibration is not always clear.

\section{$1.2 \mathrm{SU}(5)$ unification in F-theory}

The Standard Model of particle physics describes with great accuracy (up to an energy scale of few hundred $\mathrm{GeVs}$ ) all the known non-gravitational fundamental interactions of Nature; namely the electromagnetism force, the weak and the strong force. Mathematically, the Standard Model is described by a gauge theory based on the semi-simple gauge group $G_{\mathrm{SM}}=\mathrm{SU}(3) \times$ $\mathrm{SU}(2) \times U(1)$. Fundamental particles are described by irreducible representations of the gauge group. The Standard Model interactions are mediated by gauge bosons transforming in the adjoint representation of $G_{\mathrm{SM}}$ and fermionic particles (quarks and leptons) transforming under representations 
$G_{\mathrm{SM}}$ that seem at first look rather complicate and arbitrary. The Standard Model has also several unexplained properties, like for example the quantization of the electric charge, which beg for a more fundamental explanation.

In the 1970s, theoretical physicists pursued an ambitious program called "Grand Unification" with the goal of finding a simple and elegant reformulation of the Standard Model of particle physics that would make sense of its puzzling structure while providing at the same time a description of physics above its energy scale. The Standard Model being a gauge theory, representations of Lie algebras and Lie groups were the main mathematical tools for unification. This is reviewed for example in [2] or for a mathematical audience in [1]. Grand unified theories (GUTs) are based on the idea that the different interactions of the Standard Model are all unified at high energy and described by a unique gauge theory with a Lie group $G_{\mathrm{GUT}}$ containing $G_{\mathrm{SM}}$ as a subgroup. The grand unified gauge group $G_{\mathrm{GUT}}$ is usually required to be a simple group so that it depends on a unique coupling constant. A compact unified gauge group would also automatically explain the quantization of the electric charge, one of the most famous unexplained aspect of the Standard Model. A grand unified theory naturally reorganizes the Standard Model particles into fewer but larger representations. This usually implies subtle relations between apparently independent parameters of the Standard Model. It also introduces new interactions leading to prediction of new physical processes, like for example the decay of the proton (that has not yet been observed).

In the quest of the ultimate theory, string theory provides an even more ambitious program of unification since it includes a quantum description of gravity. The geometry of spacetime itself, through its extra dimensions, can geometrically generate the gauge group of a grand unified theory. The idea of geometric engineering of physical models is the source of rich dialogues between theoretical physics and mathematics. The first example of a grand unified theory was the SU(5) model proposed by Georgi and Glashow in 1974 [3]. SU(5) is the smallest simple group containing the Standard Model gauge group $G_{\mathrm{SM}}$ and able to contain the Standard Model representation for a given generation of particles. Supersymmetric versions of the GeorgiGlashow SU(5) model have been the center of important efforts to embed grand unified theories in string theory. F-theory, one of the most geometric corner of string theory, has recently played a leading role in providing phenomenological models of SU(5) unification. Describing grand unified theories by F-theory models is not only a geometrically elegant construction but is also believed to be a powerful approach to phenomenology [9]. SU(5) unification was also studied from the point of view of M-theory on manifolds with $G_{2}$ holonomies by Friedman and Witten [4]. 


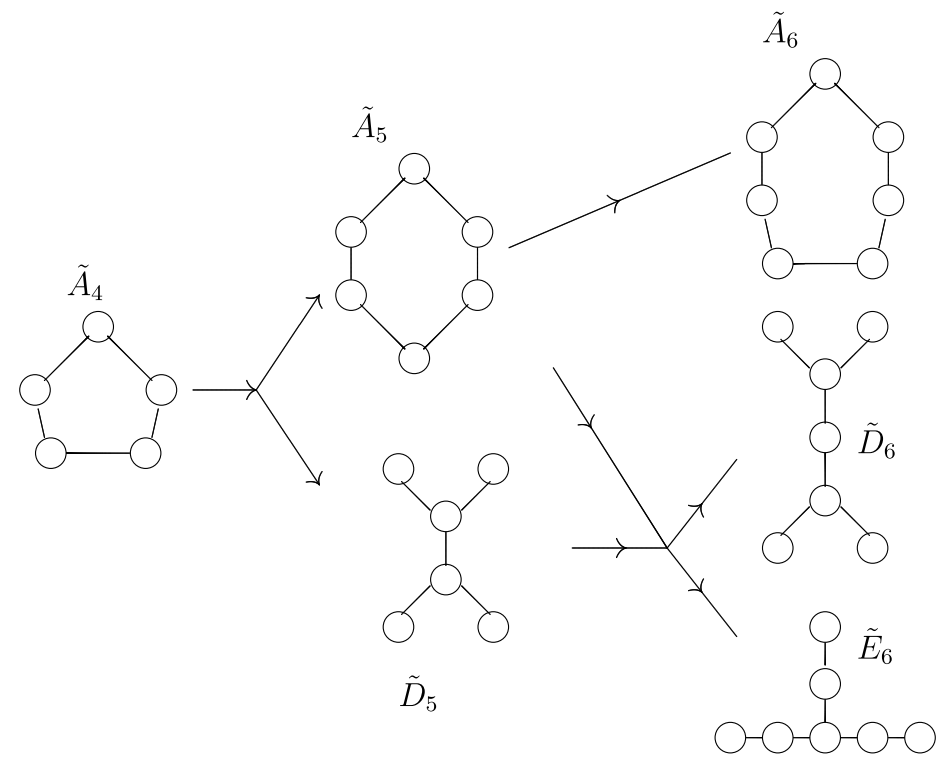

Figure 2: Conjectured singular fiber enhancements of a SU(5) GUT. Starting with codimension-one in the base, the codimension increases from left to right. Thinking in terms of dual graphs, the rank of the associated Dynkin diagrams increases as we move in codimension.

\subsection{The conjectured fiber geometry of SU(5) models}

The first step to implement an SU(5) model in F-theory is to have a component of the discriminant locus over which the elliptic fiber admits a dual graph of type split $\tilde{A}_{4}$. The "split" condition ensures that the different curves that compose the singular fiber are individually well defined without having to perform a field extension. The second step is to have the proper representations for the Standard Model fermions. It is a remarkable fact that one generation of Standard Model fermionic particles can be elegantly summarized by only two representations of SU(5), namely the complex conjugate of the fundamental representation and the second exterior power of the fundamental representation. We will denote them, respectively, as $\overline{\mathbf{5}}$ and $\mathbf{1 0}$ following the tradition in particle physics where representations are denoted by their dimensions and complex conjugation is denoted by an overall bar. In the spirit of Katz-Vafa proposal, these two representations can be, respectively, realized by the enhancement $\tilde{A}_{4} \rightarrow \tilde{A}_{5}$ and $\tilde{A}_{4} \rightarrow \tilde{D}_{5}$ over two distinct curves in the component of the discriminant over which we have the generic $\tilde{A}_{4}$ fiber. Such curves have to be singularities of the discriminant locus. One way they could occur is as intersection with a different component of the discriminant locus. Interestingly, imposing a $\tilde{A}_{4}$ fiber 
ensures that the support of the discriminant contains at least two components. Finally, the third step is to accommodate the Yukawa couplings of a (supersymmetric) SU(5) model: it requires further enhancements to $\tilde{D}_{6}$ and $\tilde{E}_{6}$ singular fibers at the points of intersections of the two matter curves. The first one $\left(\tilde{D}_{6}\right)$ gives the Yukawa couplings of the down-type quarks and the second one $\left(\tilde{E}_{6}\right)$ gives the Yukawa of the up-type quarks. There are additional important requirements that should be imposed to have a realistic model that takes care of the Higgs field, the number of generations, the breaking of the GUT group, etc. We will not consider them here. There are mostly specialization of the geometry that we will analyze. Since we are looking at the fiber geometry resulting from the condition of having a split $\tilde{A}_{4}$ structure, we will refer to the elliptic fibration simply as the $\mathrm{SU}(5)$ model.

The enhancement to $\tilde{D}_{5}, \tilde{A}_{5}$ in codimension-two and $\tilde{D}_{6}$ in codimensionthree are well understood in perturbative string theory since they can be realized by simple configurations of D-branes and orientifolds. However, the enhancement to $\tilde{E}_{6}$ necessary for the description up-type quarks Yukawa couplings does not have a description in perturbative type IIB string theory. It is a typical example of strongly coupled phenomena which requires a F-theory treatment. One of the early attractive aspect of F-theory phenomenology is the belief that these successive enhancements which are crucial for the physics are realized very naturally by the geometry of a split $\tilde{A}_{4}$. This is a highly non-trivial statement:

The discriminant should have two components, one with a singular fiber of type $\tilde{A}_{4}$ and another with a nodal curve. The intersection of these two components is composed of two curves over which the singular fiber will degenerate further to $\tilde{A}_{5}$ and $\tilde{D}_{5}$. Finally, the two curves should intersect at two different types of points leading respectively to an additional degeneration of the fiber to $\tilde{D}_{6}$ and $\tilde{E}_{6}$.

Since we assume the existence of a section, the elliptic fibration admits a birational equivalent (singular) Weierstrass model and Tate's algorithm provide a systematic way to specialize the coefficients of the Weierstrass model to impose a split $\tilde{A}_{4}$ fiber over a specific divisor of the base. The Weierstrass model will be singular, but the split $\tilde{A}_{4}$ fiber will naturally emerge after resolving its codimension-one singularities. There will be several leftover singularities in higher codimensions in the base. In the context of SU(5) unification, these extra-singularities are a blessing in disguise since they open the door to a sequence of enhancements of the singular fiber that can provide the matter fields and Yukawa couplings required for a realistic physical model. The structure of the discriminant locus of a SU(5) model has been carefully analyzed by Andreas and Curio [22]. They gave several indications 
supporting the existence of the successive enhancements demanded by SU(5) phenomenology ( $\tilde{A}_{5}$ and $\tilde{D}_{5}$ in codimension-one, $\tilde{D}_{6}$ and $\tilde{E}_{6}$ in codimensionthree). Their analysis also indicates that there might be an additional $\tilde{A}_{6}$ enhancement in codimension-three. Altogether, we have the following list of degenerations of singular fibers as we move in codimension in the base (figure 2):

$$
\tilde{A}_{4} \rightarrow\left(\tilde{A}_{5}, \tilde{D}_{5}\right) \rightarrow\left(\tilde{A}_{6}, \tilde{D}_{6}, \tilde{E}_{6}\right) .
$$

Starting from a fiber of type $\tilde{A}_{4}$, one can retrieve the same structure by assuming that all singular fibers are extended ADE Dynkin diagrams and as we move in higher codimension, the fiber enhancement is realized by increasing the rank of the dual ADE graph by one unit each time we increase the codimension by one unit. Starting from $\tilde{A}_{4}$ in codimension-one, we can then expect fibers with dual graphs $\tilde{A}_{5}$ and $\tilde{D}_{5}$ in codimension-two since they are the only extended ADE Dynkin diagrams of rank 5 . In the same spirit, we can expect dual graphs fibers with rank 6 in codimension-three, namely $\tilde{A}_{6}, \tilde{D}_{6}$ and $\tilde{E}_{6}$.

Although the fiber geometry is the backbone of any F-theory model, there has been no systematic analysis of the actual fiber structure of SU(5) models. This is mostly due to a common misconception in the physics literature according to which the fiber structure (even in higher codimension in the base) follows from Tate's algorithm. Tate's algorithm is useful to identify the singular fiber over a component of the discriminant locus of a Weierstrass model without the need to perform the explicit desingularization by just analyzing the vanishing order of the coefficients of the Weierstrass model. Unfortunately, Tate's algorithm does not apply to codimensiontwo or higher. It is easy to construct examples where a singularity of the discriminant locus does not generate a change in the fiber structure of a smooth elliptic fibration or where the change that it generates does not correspond to what is expected by "applying" Tate's algorithm. It follows that the fiber structure of $\mathrm{SU}(5)$ grand unified theories in F-theory is very much a conjecture although it is at the core of many phenomenological constructions $^{2}$. From the point of view of resolution of singularities, there is no reason to assume that the fiber structure of an SU(5) model follows the conjectured singularities: the singular elliptic fibers in codimension-two and three in the base do not have to be (extended) ADE Dynkin diagrams and the rank does not have to increase with the codimension. Since the SU(5) model has a clean definition using a (singular) Weierstrass model, studying its fiber geometry is a well posed mathematical problem that can be attacked

\footnotetext{
${ }^{2}$ For particular toric examples see [38-40].
} 
systematically by constructing an explicit desingularization. We will provide desingularizations by constructing explicit resolutions of singularities. We ask the resolution to be small (and therefore crepant) in order to preserve the Calabi-Yau condition. We also ask the resolution to preserve the flatness of the fibration in order to ensure that all fibers have the same dimension.

\subsection{Mathematical interests of the SU(5) model}

Mathematically, the conjectured fiber geometry of the SU(5) model in F-theory is an interesting structure to analyze for several reasons. Although singular fibers in codimension-one of an elliptic fibration are well understood and classified, singular fibers above higher codimension loci are only understood under conditions that are evaded by many interesting physical models. Resolution of singularities is a branch of mathematics where examples have always play a crucial role. The SU(5) model, with its sophisticated cascade of degenerations of singular fibers is a beautiful geometry to explore. As we will see along the paper, studying the details of its resolution provides a surprisingly rich geometry.

In order to put things into perspective, let us quickly review some history on singular fibers of elliptic fibrations. We will have a more detailed analysis in Section 2. In the 1960s, Kodaira [14] and Néron [15] have studied the singular fibers that can occur for an elliptic surface. Their analysis can be generalized to case of singular fibers above codimension-one points of an elliptic fibration over a higher dimensional base (see for example [19]). Miranda has studied the fiber structure of elliptic three-folds by providing a systematic method to obtain flat elliptic fibrations from (singular) Weierstrass models [16]. His results were later generalized to $n$-folds by Szydlo [17]. We will call Miranda models the elliptic fibrations with a simple normal crossing divisors obtained by Miranda (and generalized by Szydlo) by an explicit regularization of Weierstrass models. In their regularization of Weierstrass models, Miranda and Szydlo blow-up the base for example to get rid of collisions of fibers admitting different values of the $j$-invariant or to avoid collisions that do not admit small resolutions. In F-theory, the elliptic fibrations are Calabi-Yau spaces and blowing-up the base can destroy the Calabi-Yau condition depending on the dimension of the base and the dimension of the centers of the blow-up. Blowing-up the base can also add new divisors in the discriminant locus and therefore change the structure of the gauge groups and the physics of the model. We will review Miranda models and their collisions of singularities in Sections 2.3 and 2.4.

The question we address in this paper is a particular case of the following one: 
Problem 1. Consider an elliptic fibration over a complex $n$-fold, described by a (singular) Weierstrass model admitting a (split) singularity of a given Kodaira type at the generic point of a divisor of the discriminant locus and a fiber of type $I_{1}$ otherwise.

(1) Can we obtain a crepant resolution describing a flat fibration ${ }^{3}$ ?

(2) What is the tree of fiber enhancements for such a resolution?

Néron [15] has solved the problem when the base is a curve by constructing regular models for elliptic surfaces defined by Weierstrass equations. If we remove the condition to have a crepant resolution but ask to have a simple normal crossing divisor as the discriminant locus, the problem has been solved by Miranda [16] in the case of elliptic three-folds and generalized later by Zlydlo for $n$-folds [17]. Grassi and Morrison have attacked the problem for Calabi-Yau elliptic three-folds [21] under some assumptions on the type of singularities that can occur. They also discussed the F-theory interpretation of the fibers (gauge group and matter representation) while providing purely mathematical proofs. The analysis of Grassi and Morrison generalizes the results of [11] where the base is restricted to be an Hirzebruch surface $\mathbb{F}_{n}$ and the proofs rely heavily on the existence of an heterotic dual.

In this paper we treat in details the case of a split $\tilde{A}_{4}$ singularity (split $I_{5}$ ) and we assume that the base is a complex three-fold. We will construct a crepant resolution and study the resulting singular fibers. Finding one small resolution will be enough to make some strong statement. In the category of projective algebraic varieties, Batyrev proved in [20] that any two $n$-folds related by a birational map preserving their canonical class have the same Betti numbers. It follows in particular that distinct small resolutions have the same Betti numbers. Finding a small resolution of the SU(5) model might tell us important information on the conjectured fiber structure of such spaces.

\subsection{Summary of results}

The SU(5) model in F-theory is defined by the singular Weierstrass model

$$
\mathscr{E}: y^{2}+\beta_{5} x y z+\beta_{3} w^{2} y z^{2}=x^{3}+\beta_{4} w x^{2} z+\beta_{2} w^{3} x z^{2}+\beta_{0} w^{5} z^{3},
$$

where $w$ is a section of a line bundle $\mathscr{L}_{\mathrm{su}(5)}$ and $\beta_{j}(j=0,2,3,4,5)$ is a section of $\mathscr{L}^{\otimes(6-j)} \otimes \mathscr{L}_{\mathrm{su}(5)}^{\otimes(j-5)}$. This structure ensures that over a generic

\footnotetext{
${ }^{3}$ By a crepant resolution we mean that the pull back of the canonical divisor of the singular space is the canonical divisor of the smooth resolution.
} 
point of $D_{\mathfrak{s u}(5)}: w=0$, we have a singularity leading to a dual graph $\tilde{A}_{4}$ after a desingularization by blow-ups. The dual graph $\tilde{A}_{4}$ will be split: it is composed of curves that are individually well defined so that they are not subject to monodromies as we circle around the divisor $w=0$.

In this paper, we construct a crepant resolution of the SU(5) model geometry. The resolution we obtain defines a flat elliptic fibration so that all the fibers have the same dimension. The singular Weierstrass equation defining the SU(5) model admits a quite complicate discriminant, but we can resolve all the singularities in two easy steps by working directly with the Weierstrass equation. In the first step, we resolve the singularity that generates the $\tilde{A}_{4}$ fibers over a generic point of the divisor $D_{\mathfrak{s u}(5)}$. This is done by two successive blow-ups of the singular locus. The generic fiber over $D_{\mathfrak{s u}(5)}$ is then a Kodaira fiber of type $I_{5}$ with its dual graph the extended Dynkin diagram $\tilde{A}_{4}$. After the resolution of the generic singularity, there are still some left-over singularities in codimension-two and codimension-three in the base. In a second step we define a simultaneous resolution of these higher codimension singularities. One cannot just blow-up the singular locus since in codimension-two and three it will destroy the flatness of the fibration by introducing higher dimensional components on the fibers. We construct instead a small resolution by using the fact that after resolving the singularities over the codimension-one locus, the remaining singularities are described by a binomial variety. The binomial variety admits as its singular locus three lines of conifold singularities intersecting at a common point where the singularity worsen. There are six small resolutions of this geometry related to each other by a network of flop transitions that form a dihedral group $\mathrm{Dih}_{6}$ of 12 elements. These binomial transformations are induced from the symmetry of the binomial variety before the resolution. The small resolution of the binomial geometry leads to two possible types of fiber enhancement $\tilde{A}_{4} \rightarrow\left(\tilde{A}_{5}, \tilde{D}_{5}\right) \rightarrow\left(\tilde{D}_{6}, E_{6}\right)$ or $\tilde{A}_{4} \rightarrow\left(\tilde{A}_{5}, \tilde{D}_{5}\right) \rightarrow\left(\tilde{D}_{6}, E_{6}^{-}\right)$. The two possibilities differ in codimension-three by the fiber over the points where a $\tilde{E}_{6}$ enhancement is usually expected. Here we see that at these points, we have instead a projective Dynkin diagram $E_{6}$ or a fiber with dual graph $\tilde{E}_{6}^{-}:=T_{2,2,2}^{-}$. The $\tilde{E}_{6}^{-}$diagram is not $\mathrm{ADE}$ and can be better described as a $\tilde{E}_{6}$ diagram with the central node contracted to a point. The physical meaning of such fibers is not clear at all. We note that they appear at points in the base where the string coupling is of order 1, more precisely $\tau=\exp (\pi \mathrm{i} / 3)$. We also note that the fiber $E_{6}$ we obtain in codimensionthree, is really an exotic version of a $\tilde{D}_{5}$ fiber since once we remove the node that touches the section, we get a dual graph $D_{5}$. Interestingly, in the degeneration of singular fibers from codimension-two to codimension-three, the rank of the dual graph increases only for the $\tilde{D}_{6}$ fiber while in the case of the $E_{6}$ and the $\tilde{E}_{6}^{-}$fibers have there is no increase of rank. 


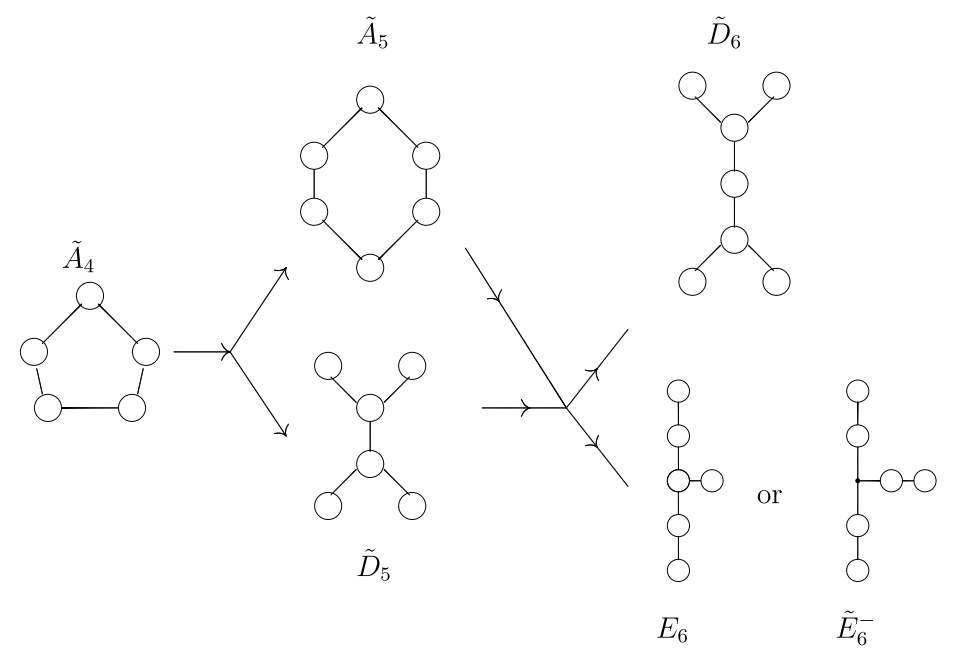

Figure 3: Degeneration of singular fibers of a small resolution of the SU(5) model. Starting with codimension-one in the base, the codimension increases from left to right. In comparison with the conjecture fiber structure, there are no fibers of type $\tilde{A}_{6}$ and the fibers of type $\tilde{E}_{6}$ are replaced by (nonKodaira) fibers of type $E_{6}$ or $\tilde{E}_{6}^{-}\left(:=T_{3,3,3}^{-}\right)$according to the choice of the resolution. We obtain six different resolutions, four of which have fibers of type $E_{6}$ in codimension-three while two have fibers of type $\tilde{E}_{6}^{-}$.

The results of this paper can then be summarized by the following propositions and the fiber degenerations are illustrated in figures 3 and 4.

Proposition 1.1 (Resolution in codimension-one). The resolution of the singularities in codimension-one is obtained by two successive blow-ups leading to a split $I_{5}$ fiber over the divisor $D_{\mathfrak{s u}(5)}$.

Proposition 1.2 (The binomial geometry). After resolving the singularities in codimension-one, we are left with higher codimensional singularities all visible in a unique patch where the variety is locally described by the following affine binomial variety in $\mathbb{C}[x, w, t, y, s]$ :

$$
y s-x w t=0,
$$

where $s=y+\beta_{5}+w \beta_{3}$ and $t=x+\beta_{4}+w \beta_{2}+w^{2} \beta_{0}$. The singular locus $y=s=x w=w t=x s=0$ of the binomial variety is composed of three lines of conifold singularities meeting at a common point where the singulary enhances. 


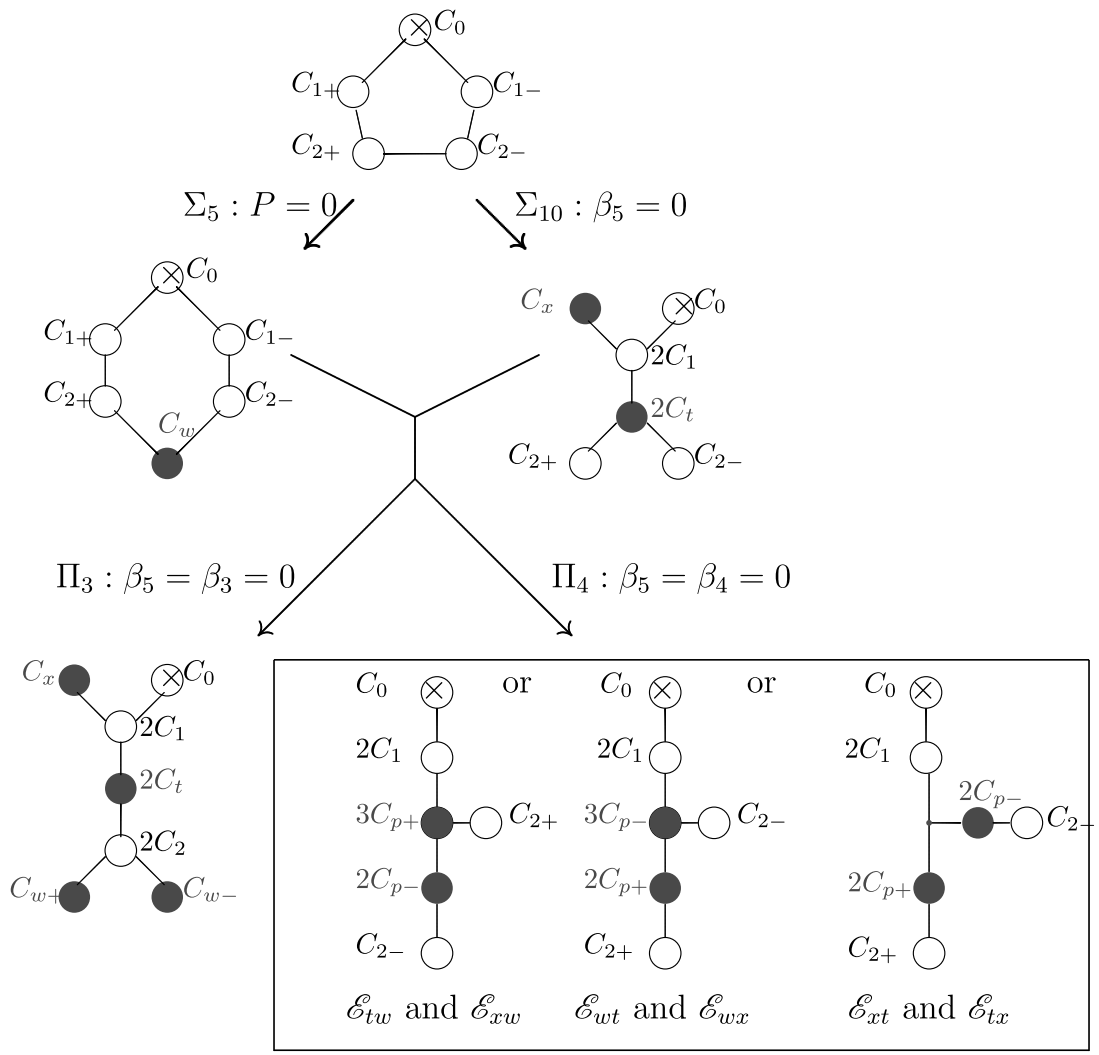

Figure 4: Fiber degeneration of a small resolution of the SU(5) model. The node $C_{0}$ is the one that touches the section. The nodes $C_{1 \pm}$ and $C_{2 \pm}$ are coming from the resolution of the singularities over a generic point of the divisor $D_{\mathfrak{s u}(5)}$. The remaining nodes $C_{x}, C_{w}$ and $C_{t}$ are obtained from the resolution of the higher codimensional singularities. We have six possible resolutions $\mathscr{E}_{x w}, \mathscr{E}_{w x}, \mathscr{E}_{x t}, \mathscr{E}_{t w}$ and $\mathscr{E}_{w t}$

Proposition 1.3 (A network of small resolutions). Using the binomial geometry, we determine six small resolutions $\left\{\mathscr{E}_{x w}, \mathscr{E}_{x t}, \mathscr{E}_{w t}, \mathscr{E}_{w x}, \mathscr{E}_{t x}, \mathscr{E}_{w t}\right\}$ :

$$
\mathscr{E}_{u_{1} u_{2}}\left\{\begin{array}{l}
u_{1} \alpha_{-}-\sigma_{-} y=0 \\
u_{2} \alpha_{+}-\sigma_{+} s=0, \\
\alpha_{-} \alpha_{+}-\sigma_{-} \sigma_{+} u_{3}=0
\end{array}\right.
$$

where $\left(u_{1}, u_{2}, u_{3}\right)$ is a permutation of $(x, w, t)$ and $\left[\alpha_{-}: \sigma_{-}\right] \times\left[\alpha_{+}: \sigma_{+}\right]$are projective coordinates of $\mathbb{F}_{0}=\mathbb{C P}^{1} \times \mathbb{C P}^{1}$. The singular fibers ${ }^{4}$ are at $w x=0$.

\footnotetext{
${ }^{4}$ This describe all the fibers above the divisor $D_{\mathfrak{s u}(5)}$ with the exception of the node $C_{0}$ that touches the section.
} 
Proposition 1.4 (Fiber enhancements in codimension-two). In codimension-two, we have an enhancement of the fiber $I_{5}$ to a fiber with dual graph $\tilde{D}_{5}$ over the curve $\Sigma_{10}: \beta_{5}=0$ and an enhancement to a fiber with dual graph $\tilde{A}_{6}$ along the curve $\Sigma_{5}: P=0$.

Proposition 1.5 (Fiber enhancements in codimension-three). In codimension-three, we have a fiber enhancement at the intersection of the two curves $\Sigma_{5}$ and $\Sigma+10$. This intersection contains two types of points that we call $\Pi_{3}$ and $\Pi_{3}\left(\Sigma_{5} \cap \Sigma_{10}=\Pi_{3} \cup \Pi_{4}\right)$ :

(1) Over $\Pi_{3}: \beta_{5}=\beta_{3}=0$, we get a fiber with dual graph $\tilde{D}_{6}$.

(2) Over $\Pi_{4}: \beta_{5}=\beta_{4}=0$, we get exotic fibers which do not increase the number of nodes of the graph. The precise dual graph of these exotic fibers depends on the choice of the resolution. For the resolutions $\mathscr{E}_{x t}$ and $\mathscr{E}_{x t}$ the fiber is of a new type that we call $\tilde{E}_{6}^{-}$while it is a projective $E_{6}$ fiber for the other four small resolutions. The fiber $\tilde{E}_{6}^{-}$is not an ADE fiber. It is a bouquet of three 2-chains intersecting at a common point.

(3) Over $\Pi_{7}: P=R=0$, there is no enhancement, the fiber is still a $\tilde{A}_{5}$.

Proposition 1.6 (Flop transitions). All the six different resolutions are connected to each other by a network of flop transitions forming a discrete dihedral group $\mathrm{Dih}_{6}$ of 12 elements.

Finally, we can rule out the existence of the conjectured resolution in a large class of varieties:

Proposition 1.7 (Birational invariance and a no-go theorem). In the category of smooth projective complex algebraic varieties, two varieties related by a birational map that does not change the canonical class have the same Betti numbers as proven by Batyrev [20]. It follows immediately that it is impossible to find a resolution of singularities that will have the same fibers as those obtained in the six small resolutions $\tilde{E}_{6}$, but will have fibers of type $\tilde{E}_{6}$ over the points $\Pi_{4}: \beta_{5}=\beta_{4}=0$ or fibers of type $\tilde{A}_{6}$ over $\Pi_{7}: P=R=0$.

If the Weierstrass model we start with is modified by specializing some of the sections $\beta_{i}$, one can end up with a very different structure of singular fibers. We are currently exploring such options. 


\subsection{Structure of the paper}

In section 2, we review several mathematical results on the structure of elliptic fibrations; Weierstrass models, Kodaira classification, Tate algorithm and Miranda models. In Section 3, we introduce in details the specific Weierstrass model that describes $\mathrm{SU}(5)$ models. We will also review the conjecture on its fiber geometry in Section 3.1. In Section 4, we resolve the singularities in codimension-one over the base and present the underlying binomial geometry in Section 5. We also discuss the toric structure of the binomial variety and study its small resolutions both torically and algebraically. We will also analyze the network of flop transitions between the different possible small resolutions we have obtained. In section 6 , we present the small resolution of the codimension-two and codimension-three singularities of the $\mathrm{SU}(5)$ by exploiting the resolution of the binomial variety. We also analyze the resulting fiber geometry. Finally we conclude in Section 7 .

\section{Elliptic fibrations and collisions of singularities}

In this section, we review some important results on elliptic fibrations and their singular fibers in different codimensions. This will put into perspective the conjectured fiber structure of SU(5) models in F-theory. In Section 2.2, we review Kodaira classification and Tate algorithm. Section 2.3 is an introduction to Miranda models. Miranda models describe certain elliptic three-folds which are flat fibrations with a restricted list of possible collisions of singular fibers. They provide (counter)examples to several common believes on the nature of colliding singularities. In particular, over loci of codimension-two or higher in the base, the singular fibers do not have to be Kodaira fibers and the rank of the singular fiber at a collision of singularities does not necessary increase, it can even decrease. We also quickly describe in Section 2.4 the generalization of Miranda models to $n$-fold by Szydlo. We will start by a quick review on elliptic curves over $\mathbb{C}$.

\subsection{Elliptic fibrations and Weierstrass models}

An elliptic curve is a smooth complex curve of genus one with a marked point on it. Topologically it is a two-torus $T^{2}=S^{1} \times S^{1}$ with a selected point. It is also an Abelian variety with the mark point as its neutral element. Modulo a similitude transformation, an elliptic curve over the complex number is always equivalent to the complex torus $\mathbb{C} /(\mathbb{Z}+\tau \mathbb{Z})$ described as the quotient of the complex plane by the double-lattice generated by 1 and the 


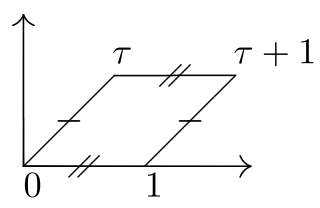

Figure 5: A torus seen as the quotient $\mathbb{C} /(\mathbb{Z}+\tau \mathbb{Z})$.

complex number $\tau$ (the period). Geometrically, the period $\tau$, characterizes the shape of the complex-torus and by convention, is restricted to live on the upper-half plane $\mathscr{H}=\{\tau \in \mathbb{C} \mid \operatorname{Im}(\tau)>0\}$. Two 2-tori are equivalent modulo similitude if their period ratios are related by a modular transformation:

$$
\tau \mapsto \frac{a \tau+b}{c \tau+d}, \quad\left(\begin{array}{ll}
a & b \\
c & d
\end{array}\right) \in \mathrm{SL}(2, \mathbb{Z}) .
$$

Using the Riemann-Roch theorem, it is easy to show that an elliptic curve can always be expressed by a cubic equation in $\mathbb{C P}^{2}$. When the characteristic is different from 2 and 3 , the cubic equation can be reduced to a (reduced) Weierstrass equation

$$
y^{2} z=x^{3}+F x z^{2}+G z^{3},
$$

where $[x: y: z]$ are the projective coordinates of $\mathbb{C P}^{2}$. We take $z=0$ to be the line at infinity. It cuts the Weierstrass equation at the rational point $[0: 1: 0]$ which is considered as the origin of the elliptic curve. The elliptic curve is regular if and only if $4 F^{3}+27 G^{2} \neq 0$. The moduli space of complex tori modulo similitudes is given by the quotient of the upper-half plane by modular transformations. There is a function, called the Klein $j$-invariant which does not change under modular transformations and maps the moduli space of complex tori modulo similitudes to the complex plane $\mathbb{C}$. Two elliptic curves are isomorphic if and only if they have the same $j$-invariant. The $j$-invariant can be computed from the Weierstrass equation as follows:

$$
j(\tau)=1728 \frac{4 F^{3}}{4 F^{3}+27 G^{2}} \quad \text { or equivalently } \quad j(\tau)=1728-\frac{27 G^{2}}{4 F^{3}+27 G^{2}} .
$$

It admits the following expression as a Laurent series:

$$
j(\tau)=\frac{1}{q}+744+\sum_{n>0} c_{n} q^{n}, \quad c_{n} \in \mathbb{N}, \quad q=\exp (2 \pi \mathrm{i} \tau) .
$$


The modular group admits as a fundamental domain the closure of the region

$$
R_{\Gamma}=\{\tau \in \mathscr{H}:|\tau+\bar{\tau}|<1 \text { and }|\tau|>1\}
$$

with a $\mathbb{Z}_{2}$ identification on the boundary given by $\tau \sim-\bar{\tau}$. When we have to make a choice between two points on the boundary, we will take the one with negative real part. It is common to use the following normalization:

$$
J(\tau)=\frac{1}{1728} j(\tau)=\frac{4 F^{3}}{4 F^{3}+27 G^{2}} .
$$

We recall some additional properties of the $J$-invariant:

$$
J(i)=1, \quad J\left(\mathrm{e}^{\frac{2 \pi}{3} \mathrm{i}}\right)=0, \quad J(-\bar{\tau})=\overline{J(\tau)}, \quad \lim _{\operatorname{Im}(\tau) \rightarrow+\infty}|J(\tau)|=\infty .
$$

If is useful to also include tori admitting an infinite value for the $j$-invariant. This corresponds to allowing an infinite value for the imaginary part of $\tau$. By the action of the modular group, we should then also include all the cusps (rational points of the real line). The $j$-invariant is then an isomorphism between the moduli space of tori (modulo similitude) and the complex sphere $\mathbb{C P}^{1}$ :

$$
J: \overline{\mathscr{H}} \rightarrow \mathbb{C P}^{1}: J(\tau) \mapsto[U, V]=\left[4 F^{3}: 4 F^{3}+27 G^{2}\right],
$$

where $[U: V]$ denotes the projective coordinates of $\mathbb{C P}^{1}$. In F-theory, since the imaginary part of $\tau$ is the inverse of the string coupling, infinite value of $\tau$ corresponds to the weak coupling limit of F-theory [10].

An elliptic fibration is a proper surjective morphism $\pi: Y \rightarrow B$ between complex varieties with connected fibers such that the general fiber is an elliptic curve. Here we consider elliptic fibrations with a smooth section. In F-theory, the section identifies the physical space seen in type IIB string theory while the elliptic fiber determines the value of the type IIB axiodilaton field. An elliptic fibration with a section is birationally equivalent to a (singular) Weierstrass model.

Let $\mathscr{L}$ be a line bundle defined over $B$. We define $E=\mathscr{O} \oplus \mathscr{L}^{\otimes(-2)} \oplus$ $\mathscr{L}^{\otimes(-3)}$, and we consider the projectization $\mathbb{P}(E)$, with tautological line bundle $\mathscr{O}(1)$. A Weierstrass model is defined as an hypersurface $Y$ of $\mathbb{P}(E)$, with equation

$$
Y: \quad z y^{2}+a_{1} x y z+a_{3} y z^{2}-\left(x^{3}+a_{2} x^{2} z+a_{4} x z^{2}+a_{6} z^{3}\right)=0 .
$$

Here $z, x$ and $y$ are sections of $\mathscr{O}(1), \mathscr{O}(1) \otimes \mathscr{L}^{\otimes(2)}$ and $\mathscr{O}(1) \otimes \mathscr{L}^{\otimes(3)}$, respectively. Each variable $a_{i}(i=1,2,3,4,6)$ is a section of the line bundle 
$\mathscr{L}^{\otimes(i)}$. One can always write the Weierstrass model in its reduced form by completing the square in $y$ and the cube in $x$ in the expression of the generalized Weierstrass model (2.8). The section of the fibration is given by $z=0$ and defines a divisor in $Y$ isomorphic to $B$. The conormal bundle of the section is isomorphic to $\mathscr{L}$. Following Deligne's formulaire [13], it is useful to introduce the following variables:

$$
\left\{\begin{array}{l}
b_{2}=a_{1}^{2}+4 a_{2}, \quad b_{4}=a_{1} a_{3}+2 a_{4}, \quad b_{6}=a_{3}^{2}+4 a_{6} \\
c_{4}=b_{2}^{2}-24 b_{4}, \quad c_{6}=-b_{2}^{3}+36 b_{2} b_{4}-216 b_{6} \\
\Delta=-\frac{1}{4} b_{2}^{2}\left(b_{2} b_{6}-b_{4}^{2}\right)-8 b_{4}^{3}-27 b_{6}^{2}+9 b_{2} b_{4} b_{6}
\end{array}\right.
$$

from which we can express the reduced Weierstrass form (2.3) with

$$
F=-\frac{1}{48} c_{4}, \quad G=-\frac{1}{864} c_{6}
$$

In terms of the reduced Weierstrass model, the discriminant $\Delta$ is simply

$$
\Delta=-16\left(4 F^{3}+27 G^{2}\right)
$$

The discriminant locus is the set of points of the base over which the elliptic fiber is not regular. An elliptic curve in the Tate's form (2.8) always admits the following $\mathbb{Z}_{2}$ symmetry, which corresponds to the inverse law of the torus group [13]:

$$
(x, y) \mapsto\left(x,-y-a_{1} x-a_{3}\right)
$$

This involution plays an central role in the description of orientifolds in F-theory $[10,26-28]$. It is related to the discrete operator $(-1)^{F_{\mathrm{L}}} \Omega$ defined in perturbative string theory. Here $F_{\mathrm{L}}$ is the left-hand fermion number and $\Omega$ is the worldsheet parity inversion. From the point of view of the modular group, it is just minus the identity $\left(-I_{2}\right)$ and acts trivially on the period [10].

\subsection{Kodaira classification and Tate algorithm}

In the early 1960s, Kodaira has classified singular fibers of (minimal) elliptic surfaces [14]. Kodaira's classification can be extended to singular fibers over codimension-one points in the base modulo mild assumptions (table 2). He identified eight types of singular fibers, including two infinite series 
$\left(I_{1}, I_{n}(n>0)\right.$, II, III, IV,$I_{n}^{*}$, II*, $\left.\mathrm{III}^{*}\right)$. Each fiber is constituted of intersecting rational curves and the dual graph of such singular fibers are closely related to simply-laced (affine) Dynkin diagrams. In Kodaira list, there are only two irreducible singular fibers: nodal curves (type $I_{1}$ ) and cusp (type II). All the remaining fibers are reducible. There are three that are not extended ADE diagrams: type $I_{2}$ is composed of two rational curves meeting transversally at two points, type III consists of two rational curves tangent at a point and type IV is composed of three rational curves meeting transversally at a common point (type IV). The remaining types are extended ADE Dynkin diagram: the dual graph $\tilde{A}_{n}$ gives type $I_{n}(n \leq 3)$, the dual graph $\tilde{D}_{4+n}$ corresponds to type $I_{n}^{*}$, the dual graphs $\tilde{E}_{6}, \tilde{E}_{7}$ and $\tilde{E}_{8}$ correspond, respectively, to type IV*, III* and II*. In 1964, André Néron has reproduced Kodaira's list by explicitly constructing regular models for elliptic surfaces defined by Weierstrass models [15]. In 1975, Tate, using Néron results, has developed an algorithm which gives the type of singular fibers of an elliptic surface by manipulating the coefficients of the Weierstrass equation [18].

In F-theory, Tate's algorithm has been refined to determine the gauge groups (even non-simply laced ones) associated to seven-branes wrapping divisors of the discriminant locus [11,37] (table 3). Non-simply laced Dynkin diagrams are included in a Kodaira fibers those nodes that are exchanged by the action of monodromies. Some specific examples also extended Tate's algorithm to certain codimension-two loci in order to predict the matter contents at the intersection of two divisors of the discriminant locus. Unfortunately, in the physics literature, Tate's algorithm is often used outside of its domain of validity. This has created important misconceptions on the structure of singular fibers over points located in higher codimensions in the base.

\subsection{Miranda models and collisions of singular fibers}

Singular fibers of an elliptic fibration over loci in codimension-two or higher codimension in base are not well understood, except when strong conditions like simple normal crossing of the discriminant locus and preservation of the $j$-invariant at collisions are imposed. Under such conditions, Miranda has analyzed in 1983 the singular fibers for elliptic three-folds defined by Weierstrass models [16]. His construction was generalized to $n$-folds by Szydlo in 1999 [17]. Miranda showed that it is always possible to obtain a regular model for an elliptic three-fold defined by a Weierstrass equation. We will refer to such elliptic fibrations as Miranda models. The strategy of Miranda consists of blowing-up the base over intersections of divisors that 
Table 2: Kodaira classification of singular fibers of an elliptic fibration. The fiber of type $I_{0}^{*}$ is special among its family $I_{n}^{*}$ because its $J$-invariant can take any value in $\mathbb{C}$. A fiber of type $I_{n}$ or $I_{n}^{*}(n>0)$ has a pole of order $n$ over the divisor on which it is defined.

\begin{tabular}{lccccccc}
\hline Type $\operatorname{ord}(F)$ & $\operatorname{ord}(G)$ & $\operatorname{ord}(\Delta)$ & $J$ & Monodromy & Fiber \\
\hline$I_{0}$ & $\geq 0$ & $\geq 0$ & 0 & $\mathbb{C}$ & $\mathrm{I}_{2}$ & Smooth torus \\
$I_{1}$ & 0 & 0 & 1 & $\infty$ & $\left(\begin{array}{cc}1 & 1 \\
0 & 1\end{array}\right)$ & (Nodal curve) \\
$I_{n}$ & 0 & 0 & $n>1$ & $\infty$ & $\left(\begin{array}{cc}1 & n \\
0 & 1\end{array}\right)$ &
\end{tabular}


Table 3: F-theory Tate's algorithm. The superscript (s/ns/ss) stands for (split/non-split/semi-split), meaning that (there is/there is not/ there is a partial) monodromy action by an outer automorphism on the vanishing cycles along the singular locus.

\begin{tabular}{|c|c|c|c|c|c|c|c|}
\hline Type & Group & $a_{1}$ & $a_{2}$ & $a_{3}$ & $a_{4}$ & $a_{6}$ & $\Delta$ \\
\hline$\overline{I_{0}}$ & - & 0 & 0 & 0 & 0 & 0 & 0 \\
\hline$I_{1}$ & - & 0 & 0 & 1 & 1 & 1 & 1 \\
\hline$I_{2}$ & $\mathrm{SU}(2)$ & 0 & 0 & 1 & 1 & 2 & 2 \\
\hline$I_{3}^{\mathrm{ns}}$ & unconven. & 0 & 0 & 2 & 2 & 3 & 3 \\
\hline$I_{3}^{\mathrm{s}}$ & unconven. & 0 & 1 & 1 & 2 & 3 & 3 \\
\hline$I_{2 k}^{\mathrm{ns}}$ & $\mathrm{Sp}(\mathrm{k})$ & 0 & 0 & $k$ & $k$ & $2 k$ & $2 k$ \\
\hline$I_{2 k}^{\mathrm{s}}$ & $\mathrm{SU}(2 k)$ & 0 & 1 & $k$ & $k$ & $2 k$ & $2 k$ \\
\hline$I_{2 k+1}^{\mathrm{ns}}$ & unconven. & 0 & 0 & $k+1$ & $k+1$ & $2 k+1$ & $2 k+1$ \\
\hline$I_{2 k+1}^{\mathrm{S}}$ & $\mathrm{SU}(2 k+1)$ & 0 & 1 & $k$ & $k+1$ & $2 k+1$ & $2 k+1$ \\
\hline II & - & 1 & 1 & 1 & 1 & 1 & 2 \\
\hline III & $\mathrm{SU}(2)$ & 1 & 1 & 1 & 1 & 2 & 3 \\
\hline $\mathrm{IV}^{\mathrm{ns}}$ & unconven. & 1 & 1 & 1 & 2 & 2 & 4 \\
\hline $\mathrm{IV}^{\mathrm{s}}$ & $\mathrm{SU}(3)$ & 1 & 1 & 1 & 2 & 3 & 4 \\
\hline$I_{0}^{* \mathrm{~ns}}$ & $G_{2}$ & 1 & 1 & 2 & 2 & 3 & 6 \\
\hline$I_{0}^{* s s}$ & $\mathrm{SO}(7)$ & 1 & 1 & 2 & 2 & 4 & 6 \\
\hline$I_{0}^{* \mathrm{~s}}$ & $\mathrm{SO}(8)^{*}$ & 1 & 1 & 2 & 2 & 4 & 6 \\
\hline$I_{1}^{* \mathrm{~ns}}$ & $\mathrm{SO}(9)$ & 1 & 1 & 2 & 3 & 4 & 7 \\
\hline$I_{1}^{* \mathrm{~s}}$ & $\mathrm{SO}(10)$ & 1 & 1 & 2 & 3 & 5 & 7 \\
\hline$I_{2}^{* \mathrm{~ns}}$ & $\mathrm{SO}(11)$ & 1 & 1 & 3 & 3 & 5 & 8 \\
\hline$I_{2}^{* \mathrm{~s}}$ & $S O(12)^{*}$ & 1 & 1 & 3 & 3 & 5 & 8 \\
\hline$I_{2 k-3}^{* \mathrm{nn}}$ & $\mathrm{SO}(4 k+1)$ & 1 & 1 & $k$ & $k+1$ & $2 k$ & $2 k+3$ \\
\hline$I_{2 k-3}^{* \mathrm{~s}}$ & $\mathrm{SO}(4 k+2)$ & 1 & 1 & $k$ & $k+1$ & $2 k+1$ & $2 k+3$ \\
\hline$I_{2 k-2}^{* \mathrm{~ns}}$ & $\mathrm{SO}(4 k+3)$ & 1 & 1 & $k+1$ & $k+1$ & $2 k+1$ & $2 k+4$ \\
\hline$I_{2 k-2}^{* \mathrm{~s}}$ & $\mathrm{SO}(4 k+4)^{*}$ & 1 & 1 & $k+1$ & $k+1$ & $2 k+1$ & $2 k+4$ \\
\hline $\mathrm{IV}^{* \mathrm{~ns}}$ & $F_{4}$ & 1 & 2 & 2 & 3 & 4 & 8 \\
\hline $\mathrm{IV}^{* \mathrm{~s}}$ & $E_{6}$ & 1 & 2 & 2 & 3 & 5 & 8 \\
\hline III* & $E_{7}$ & 1 & 2 & 3 & 3 & 5 & 9 \\
\hline $\mathrm{II}^{*}$ & $E_{8}$ & 1 & 2 & 3 & 4 & 5 & 10 \\
\hline Non-min & - & 1 & 2 & 3 & 4 & 6 & 12 \\
\hline
\end{tabular}


would lead to "bad collisions" of singularities, then pull-back the fibrations to the new base and he then desingularizes the total space. After enough blow-ups, the discriminant locus is a simple normal crossing divisor with only a very limited number of collisions admitting small resolutions. A Miranda model only has the following eight collisions:

$$
\begin{aligned}
& J=\infty \quad: \quad I_{n}+I_{m} \quad \rightarrow \quad I_{n+m} \\
& I_{2 n}+I_{m}^{*} \quad \rightarrow \quad I_{n+m}^{*} \\
& I_{2 n+1}+I_{m}^{*} \rightarrow I_{n+m+1}^{*+} \text { (1) (a total of } n+m+5 \text { nodes) } \\
& J=0 \quad: \quad I I+I V \quad \longrightarrow \quad \text { (1)-2 } \\
& I I+I_{0}^{*} \quad \longrightarrow \text { (1)-(2) } \\
& I V+I_{0}^{*} \quad \longrightarrow \text { (1)-(2)-(3)-(4)-2 } \\
& I I+I V^{*} \longrightarrow \text { (1)-2)(4)-2 } \\
& J=1 \quad: \quad I I I+I_{0}^{*} \quad \longrightarrow \text { (1)-2(3)-(2)-(1) }
\end{aligned}
$$

Only the first two collisions (namely $I_{n}+I_{m}$ and $I_{2 n}+I_{m}^{*}$ ) leads to Kodaira fibers. The others are not Kodaira fibers but admit $D_{k}$ and $A_{k}$ projective Dynkin diagrams as dual graphs. The new fiber of type $I_{n}^{*+}$ has the shape of a diagram of a projective Dynkin diagram of type $D_{n+4}$. We summarize all the allowed collisions of Miranda's models in table 4. The last column of that table shows the fiber that would have been obtained if one had resolved an elliptic surface with base a general smooth curve passing through the collision. It illustrates that abusing Tate's algorithm already in codimension-two leads to a misinterpretation of the fiber structure and that the rank of the ADE fiber does not necessary increase at a collision, it can actually decrease.

\subsection{Szydlo generalization of Miranda models}

Assuming the same conditions as Miranda, Szydlo has analyzed the general case of collisions in higher codimensions. Interestingly, starting from codimension-three, the only collisions possible are those with $J=\infty$ ( type 
Table 4: Colliding singularities in an elliptic three-fold as constructed by Miranda. The non-Kodaira fiber $I_{n}^{*+}$ has the shape of a diagram of type $D_{n+4}$. The last column shows the fiber that would be obtained for an elliptic with base a smooth curve passing through the point of collision.

\begin{tabular}{|c|c|c|c|}
\hline$j$-inv & Collision & Dual graph & $\begin{array}{l}\text { If the base was a smooth curve } \\
\text { through the collision point }\end{array}$ \\
\hline$\infty$ & $I_{M_{1}}+I_{M_{2}}$ & $\begin{array}{l}\text { (1)-(1)-(1)-(1)-... (1) } \\
\text { (1)-(1)-(1)- - (1) } \\
I_{M_{1}+M_{2}}\end{array}$ & same \\
\hline$\infty$ & $I_{2 n}+I_{m}^{*}$ & (1) $I_{n+m}^{\star}$ & $1_{2 n+m}^{\star}$ \\
\hline$\infty$ & $I_{2 n+1}+I_{m}^{*}$ & $\begin{array}{l}\text { (1) } I_{n+m}^{*+} \\
(n+m+4) \text { components }\end{array}$ & (1) $I_{2 n+m+1}^{\star}$ \\
\hline 0 & $\mathrm{II}+\mathrm{IV}$ & (1)-2) & $\begin{array}{c}1 \text { (1)-1) } \\
I_{0}^{\star} \quad 1 \\
\text { (1)-(2)-(3)-(1) }\end{array}$ \\
\hline 0 & $\mathrm{II}+I_{0}^{*}$ & (1)-(2)-3) & \\
\hline 0 & $\mathrm{II}+\mathrm{IV}^{*}$ & (1)-(2)-(3)-(4)-(2) & $I^{\star}$ \\
\hline 0 & $\mathrm{IV}+I_{0}^{*}$ & (1)-(2)-(4)-2) & \\
\hline 1728 & $\mathrm{III}+I_{0}^{*}$ & (1)-(2)-(3)-(2)-(1) & III $^{\star}$ \\
\hline
\end{tabular}

$I_{n}$ and $I_{n}^{*}$ ) with the following restrictions: there are at most one fiber of type $I_{n}^{*}$ and at most one fiber of type $I_{2 m+1}$, the number of fiber of type $I_{2 n}$ is bounded by the codimension of the collision. Taking this into account we 
have the following four types of collisions:

$$
\begin{array}{rlr}
J=\infty: & I_{2 n_{1}}+\cdots I_{2 n_{k}} & \longrightarrow I_{2 n}, \quad n= \\
& I_{2 n_{1}}+\cdots I_{2 n_{k}}+I_{2 r+1} & \longrightarrow I_{2 n+2 r+1}, \\
& I_{2 n_{1}}+\cdots I_{2 n_{k}}+I_{m}^{*} & \longrightarrow I_{n+m+1}^{*}, \\
& I_{2 n_{1}}+\cdots I_{2 n_{k}}+I_{2 r+1}+I_{m}^{*} & \longrightarrow I_{n+r+m+1}^{*+} .
\end{array}
$$

The resolution of the singularities at the collisions depends on some discrete choices. In particular, the order in which the blow-ups are performed is crucial for the final result. For example, Miranda and Szydlo do not have the same results for the collision IV $+I_{0}^{*}$ and the justification can be traced back to different conventions on how to order the blow-ups:

\begin{tabular}{c|c} 
(Miranda) & (Szydlo) \\
\hline$I V+I_{0}^{*}:$ (1)-(2)-(4)-(2) & $I_{0}^{*}+I V:$ (1)-(2)-(3)-(2)
\end{tabular}

\section{$2.5 \quad$ F-theory versus Miranda models}

The results of Miranda and Szydlo provide an algorithm to obtain regular models from singular Weierstrass models satisfying some conditions like simple normal crossing for the discriminant locus. Miranda models have been used in F-theory in cases where blowing-up the base did not destroy the Calabi-Yau condition [32]. However, for applications of F-theory to grand unification, the assumptions of Miranda and Szydlo are very naturally violated $^{5}$. For example, the enhancement $\tilde{D}_{5} \rightarrow \tilde{E}_{6}$ which is very natural in the context of grand unified theories is not allowed within Miranda or Szydlo's framework since it involves fiber of different $J$-invariant $(J=\infty \rightarrow J=0)$. Even the transition $\tilde{A}_{4} \rightarrow \tilde{D}_{5}$, which is allowed in perturbative type IIB, is not allowed in a Miranda's collision since the only fiber of type $I_{n}^{*}$ that can be obtained in codimension-two have $n \geq 2$. These examples illustrate that in F-theory, one has to include wilder singularities in the discriminant locus and relax the requirement of a well behaved $J$-invariant. But at the same time, one has to be careful with blowing-up the base since it can lead to violation of the Calabi-Yau condition. Finally, we would like to point

\footnotetext{
${ }^{5}$ Even in a purely geometric context one can argue that asking for normal crossing is too strong since the discriminant locus of a general Weierstrass model has always a cusp singularity and this is not really an invitation for normal crossing. But the condition of normal crossing often occurs in mathematics since it makes life easier in proving theorems. However, in physics, one does not always have the freedom to choose his assumptions.
} 
out that even the flatness condition is not required in F-theory. Flatness of the fibration is equivalent here to the requirement that all the fibers are of pure dimension one. But there are interesting physical phenomena when a fiber contains a higher dimensional component [31]. We have seen that the assumption of flatness, normal crossing and well defined $J$-invariant would eliminate interesting physics. However, once we remove these very friendly conditions, it is not clear how one will achieve a classification at all.

\section{Geometric engineering of SU(5) models in F-theory}

In Section 2 we have reviewed the basic notions of elliptic fibrations. In this short section, we will present the construction of the $\mathrm{SU}(5)$ model in F-theory and introduce formally the conjecture on its singular fibers.

An SU(5) grand unified theory is geometrically engineered in F-theory by a Weierstrass model admitting a split $I_{5}$ fiber over a general point of a divisor of the discriminant locus:

$$
\text { split } I_{5} \Longrightarrow \mathrm{SU}(5) \text { GUT. }
$$

We recall that a Weierstrass model $Y \rightarrow B$ over the base $B$ is given by the following cubic equation written in a $\mathbb{P}^{2}$-bundle over the base $B$ :

$$
z y^{2}+a_{1} x y z+a_{3} y z^{2}=x^{3}+a_{2} x^{2} z+a_{4} x z^{2}+a_{6} z^{3},
$$

where the variables $x, y$ and $z$ are the projective coordinates of a $\mathbb{P}^{2}$-bundle. Each variable $a_{i}(i=1,2,3,4,6)$ is a section of the line bundle $\mathscr{L}^{\otimes(-i)}$. Suppose that $D_{\mathfrak{s u}(5)}$ is the divisor over which we want to have a split $I_{5}$ singular fiber. We will assume that $D_{\mathfrak{s u}(5)}$ is the zero locus of a section $w$ of a line bundle $\mathscr{L}_{\mathfrak{s u}(5)}$ :

$$
D_{\mathfrak{s u}(5)}: \quad w=0 .
$$

We can now consider the coefficients of the Weierstrass equation as polynomials in $w$. It follows from table 3 that after resolving the singularity, we will have a split $I_{5}$ fiber over $w=0$ if we impose the following specialization of the coefficients $a_{i}$ :

$$
a_{1}=\beta_{5}, \quad a_{2}=\beta_{4} w, \quad a_{3}=\beta_{3} w^{2}, \quad a_{4}=\beta_{2} w^{3}, \quad a_{6}=\beta_{0} w^{5} .
$$

Here, each $\beta_{j}(j=0,2,3,4,5)$ is a section of the line bundle $\mathscr{L}^{\otimes(6-j)} \otimes$ $\mathscr{L}_{\mathfrak{s u}(5)}^{\otimes(j-5)}$. We assume each $\beta_{j}$ does not vanish identically over $w=0$. The 
resulting (singular) Weierstrass model is given by the following equation:

$$
\mathscr{E}: z y^{2}+\beta_{5} x y z+\beta_{3} w^{2} y z^{2}-\left(x^{3}+\beta_{4} w x^{2} z+\beta_{2} w^{3} x z^{2}+\beta_{0} w^{5} z^{3}\right)=0,
$$

where $w$ and $\beta_{j}(j=0,2,3,4,5)$ are, respectively, sections of $\mathscr{L}_{\mathfrak{s u}(5)}$ and $\mathscr{L}^{\otimes(6-j)} \otimes \mathscr{L}_{\mathfrak{s u}(5)}^{\otimes(j-5)}$. The Weierstrass model admits the following factorization of its discriminant:

$$
\Delta=-w^{5} \Delta^{\prime}
$$

The discriminant locus is the union of two divisors, namely $D_{\mathfrak{s u}(5)}: w=0$ and $D: \Delta^{\prime}=0$ where

$$
\Delta^{\prime}=\beta_{5}^{4} P+w \beta_{5}^{2}\left(8 \beta_{4} P+\beta_{5} R\right)+w^{2}\left(16 \beta_{3}^{2} \beta_{4}^{3}+\beta_{5} Q\right)+w^{3} S+w^{4} T+w^{5} U .
$$

Here $P, R, Q, S, T$ and $U$ are polynomials in $\beta_{j}(j=0,2,3,4,5)$ :

$$
\begin{aligned}
P= & \beta_{3}^{2} \beta_{4}-\beta_{2} \beta_{3} \beta_{5}+\beta_{0} \beta_{5}^{2}, \quad R=4 \beta_{0} \beta_{4} \beta_{5}-\beta_{3}^{3}-\beta_{2}^{2} \beta_{5}, \\
Q= & -2\left(18 \beta_{3}^{3} \beta_{4}+8 \beta_{2} \beta_{3} \beta_{4}^{2}-15 \beta_{2} \beta_{3}^{2} \beta_{5}+4 \beta_{2}^{2} \beta_{4} \beta_{5}-24 \beta_{0} \beta_{4}^{2} \beta_{5}+18 \beta_{0} \beta_{3} \beta_{5}^{2}\right), \\
S= & 27 \beta_{3}^{4}-72 \beta_{2} \beta_{3}^{2} \beta_{4}-16 \beta_{2}^{2} \beta_{4}^{2}+64 \beta_{0} \beta_{4}^{3}+96 \beta_{2}^{2} \beta_{3} \beta_{5}-144 \beta_{0} \beta_{3} \beta_{4} \beta_{5} \\
& -72 \beta_{0} \beta_{2} \beta_{5}^{2}, \\
T= & 8\left(8 \beta_{2}^{3}+27 \beta_{0} \beta_{3}^{2}-36 \beta_{0} \beta_{2} \beta_{4}\right), \quad U=432 \beta_{0}^{2} .
\end{aligned}
$$

The reduced Weierstrass model corresponding to equation (3.4) is given by

$$
\begin{aligned}
c_{4}= & -48 w^{3} \beta_{2}+16 w^{2} \beta_{4}^{2}-24 w^{2} \beta_{3} \beta_{5}+8 w \beta_{4} \beta_{5}^{2}+\beta_{5}^{4}, \\
c_{6}= & -864 w^{5} \beta_{0}-216 w^{4} \beta_{3}^{2}+288 w^{4} \beta_{2} \beta_{4}-64 w^{3} \beta_{4}^{3} \\
& +\beta_{5}\left(144 w^{3} \beta_{3} \beta_{4}+72 w^{3} \beta_{2} \beta_{5}-48 w^{2} \beta_{4}^{2}+36 w^{2} \beta_{3} \beta_{5}-12 w \beta_{4} \beta_{5}^{2}-\beta_{5}^{5}\right) .
\end{aligned}
$$

\subsection{Conjectured fiber geometry}

The support of the discriminant locus $\Delta$ of the elliptic fibration that we have introduced to describe an SU(5) GUT is the union of two divisors, namely $D_{\mathfrak{s u}(5)}: w=0$ (of multiplicity 5 ) and $D^{\prime}: \Delta^{\prime}=0$ (of multiplicity 
one). Over a general point of $D_{\mathfrak{s u}(5)}$, we have a split $I_{5}$ fiber corresponding to a $\mathrm{SU}(5)$ gauge group. Over a general point of $D^{\prime}$ we have a $I_{1}$ fiber (a nodal curve):

$$
D_{\mathfrak{s u}(5)}: w=0 \quad\left(I_{5}\right), \quad D^{\prime}: \Delta^{\prime}=0 \quad\left(I_{1}\right) .
$$

Since in general $\Delta^{\prime}$ does not factorize further, we have five D7-branes wrapping the divisor $D_{\mathfrak{s u}(5)}: w=0$ and one D7-brane wrapping the divisor $D^{\prime}: \Delta^{\prime}=0$. The divisor $D^{\prime}$ is actually singular and all its singularities are supported on the codimension-two locus $w=P \beta_{5}=0$ which is the union of two curves of $D_{\mathfrak{s u}(5)}$, namely $\Sigma_{5}: w=P=0$ and $\Sigma_{10}: w=\beta_{5}=0$. Interestingly, the singular locus of $D^{\prime}$ is exactly its intersection with $D_{\mathfrak{s u}(5)}$. We have

$$
D_{\mathfrak{s u}(5)} \cap D^{\prime}=\Sigma_{5} \cup \Sigma_{10} .
$$

It is conjectured that the $I_{5}$ fiber enhances to a $I_{6}$ fiber over $\Sigma_{5}$ and to a $I_{1}^{*}$ fiber over $\Sigma_{10}$ (see for example $[22,29]$ ):

$$
\Sigma_{5}: w=P=0 \quad\left(I_{6} ?\right), \quad \Sigma_{10}: w=\beta_{5}=0 \quad\left(I_{1}^{*} ?\right) .
$$

In codimension-three, at the intersection of $\Sigma_{5}$ and $\Sigma_{10}$ the singularity is expected to enhance further. The intersection of $\Sigma_{5}$ and $\Sigma_{10}$ is the union of two codimension-three loci in the base, $\Pi_{3}$ and $\Pi_{4}$ :

$$
\Sigma_{5} \cap \Sigma_{10}=\Pi_{3} \cup \Pi_{4} .
$$

Over $\Pi_{3}$ and $\Pi_{4}$, it is conjectured that the fiber enhances to a fiber $I_{2}^{*}$ and $\mathrm{IV}^{*}$ (or more generally to a fiber with dual graph $\tilde{D}_{6}$ and $\tilde{E}_{6}$ ):

$$
\Pi_{3}: w=\beta_{3}=\beta_{5}=0 \quad\left(I_{2}^{*} ?\right) \cup \Pi_{4}: w=\beta_{4}=\beta_{5}=0 \quad\left(I V^{*} ?\right) .
$$

The curve $\Sigma_{5}$ contains additional points $\Pi_{7}: w=P=R=0$ over which the singular fiber $I_{6}$ is conjectured to enhance further to a fiber of type $I_{7}[22,29]$ :

$$
\Pi_{7}: w=P=R=0 \quad\left(I_{7} ?\right) .
$$

These points are also codimension-three points in the base. These are not coming from the collisions of two curves, but they contain as a proper subset the points $\Pi_{3}$. The conjectured tree of singular fibers enhancement is summarized in figure 6 and table 7 (see table 5). 


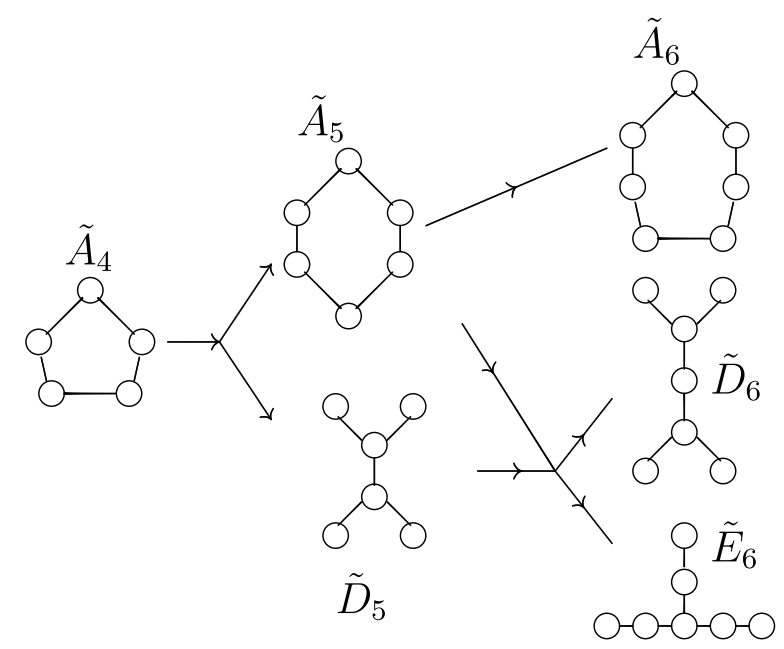

Figure 6: Conjectured singular fiber enhancements of a SU(5) GUT. Starting with codimension-one in the base, the codimension increases from left to right. Thinking in terms of the dual graph, the rank of the associated Dynkin diagram increases by 1 as we move in codimension.

\section{Resolution over codimension-one loci in the base}

In this section we resolve the singularities that project to codimension-one loci in the base. The variety $\mathscr{E}$ is singular at $x=y=w=0$. Fiberwise, we have a singular point on the elliptic fiber above the divisor $D_{\mathfrak{s u}(5)}: w=0$ in the base. The divisor $D_{\mathfrak{s u}(5)}$ is a component of the discriminant locus of multiplicity 5 . The resolution is obtained by two successive blow-ups after which the singular fiber is replaced by a cycle of five rational curves defining the Kodaira type $I_{5}$ over $D_{\mathfrak{s u}(5)}$.

\subsection{Blowing-up the codimension-one singularity}

Our starting point is the defining Weierstrass equation of the SU(5) GUT model:

$$
\mathscr{E}: z y^{2}+\beta_{5} x y z+\beta_{3} w^{2} y z^{2}-\left(x^{3}+\beta_{4} w x^{2} z+\beta_{2} w^{3} x z^{2}+\beta_{0} w^{5} z^{3}\right)=0 .
$$

The support of all the singularities of the variety $\mathscr{E}$ is

$$
\operatorname{Sing}(\mathscr{E}): \quad x=y=w=0 .
$$


Table 5: Conjectured singular fiber enhancements of a general SU(5) GUT model.

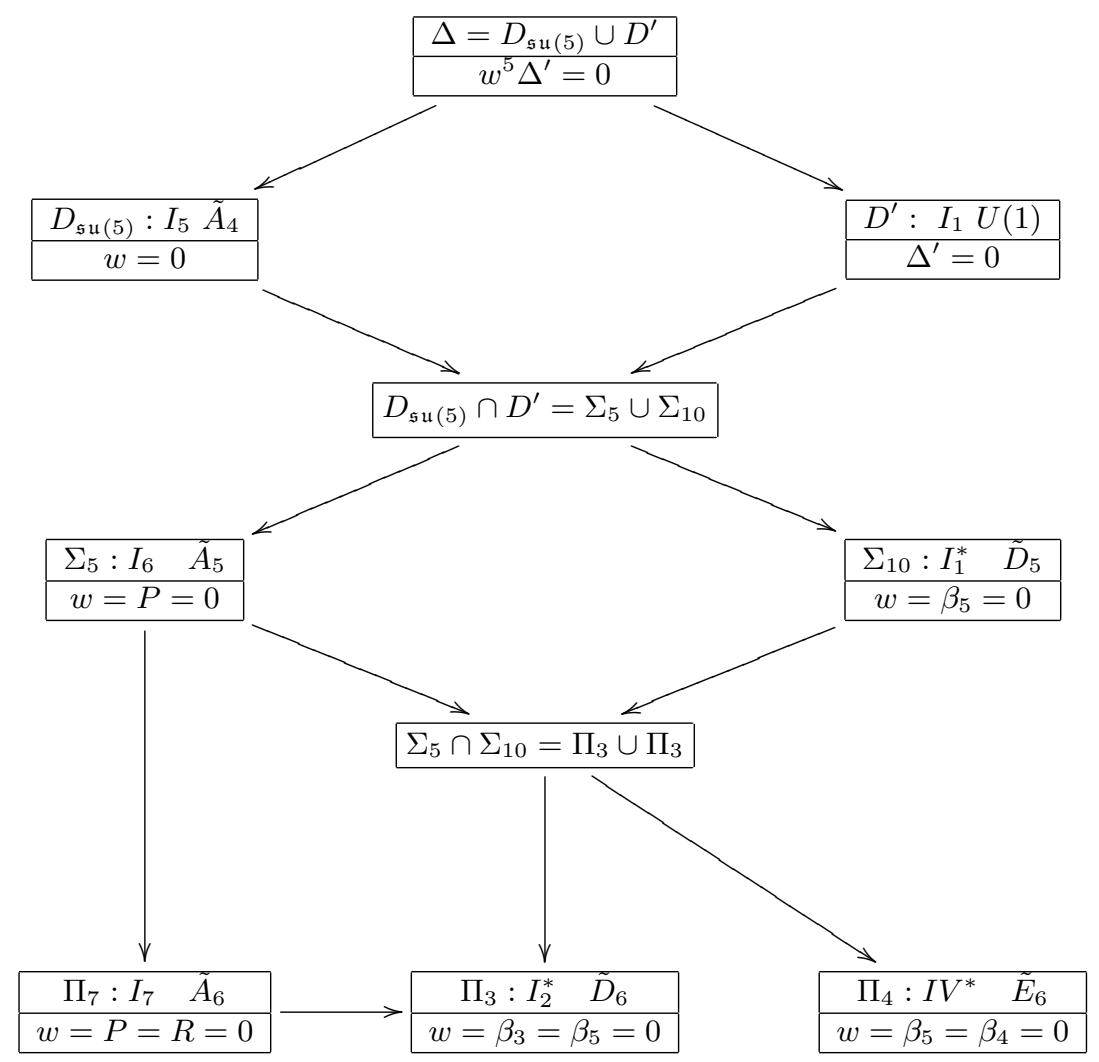

Over the divisor $D_{\mathfrak{s u}(5)}: w=0$, the elliptic fiber degenerates into the nodal curve

$$
C_{0}: z y^{2}+\beta_{5} x y z-x^{3}=0 .
$$

This nodal curve degenerates further to a cuspidial curve over the codimension-two locus $\beta_{5}=w=0$ of the base.

We will blow-up this singular locus $x=y=w=0$. For that matter we introduce the projective coordinates $\left[U_{1}, U_{2}, U_{3}\right]$ of a $\mathbb{C P}^{2}$ together with the following relations:

$$
x U_{2}=y U_{1}, \quad x U_{3}=w U_{1}, \quad y U_{3}=w U_{2} .
$$

Working first in a patch defined $\mathscr{U}_{1}$ defined by $U_{1} \neq 0$, we have $y=\frac{U_{2}}{U_{1}} x$, $w=\frac{U_{3}}{U_{1}} x$. Defining $x_{1}=x, y_{1}=U_{2} / U_{1}$ and $w_{1}=U_{3} / U_{1}$, the blow-up can 
be expressed in $\mathscr{U}_{1}$ by the morphism $\varphi_{1}: \quad(x, y, w) \mapsto\left[x_{1}, x_{1} y_{1}, x_{1} w_{1}\right]$. We can proceed in a similar way in the patches $\mathscr{U}_{2}$ and $\mathscr{U}_{3}$ defined respectively by $U_{2} \neq 0$ and $U_{3} \neq 0$. Altogether, the blow-up is computed using the three morphisms:

$$
\begin{array}{lll}
\varphi_{1}: & (x, y, w) \mapsto\left[x_{1}, x_{1} y_{1}, x_{1} w_{1}\right], \\
\varphi_{2}: & (x, y, w) \mapsto\left[y_{2} x_{2}, y_{2}, y_{2} w_{2}\right], \\
\varphi_{3}: & (x, y, w) \mapsto\left[w_{3} x_{3}, w_{3} y_{3}, w_{3}\right] .
\end{array}
$$

The different patches are glued together along their intersection by requiring the morphism to match. For example, along $\mathscr{U}_{1} \cap \mathscr{U}_{2}$, the gluing is based on matching $\varphi_{1}=\varphi_{2}$ and gives $\left(x_{1}, y_{1}, w_{1}\right)=\left(x_{2} y_{2}, 1 / x_{2}, w_{2} / x_{2}\right)$. Before the blow-up, the special fiber is obtained by cutting $\mathscr{E}$ along $w=0$. After the blow-up, we have to keep track of the fiber function in every patch. In the patch $\mathscr{U}_{1}, \mathscr{U}_{2}$ and $\mathscr{U}_{3}$, we get, respectively, the fiber function $w_{1} x_{1}$, $w_{2} y_{2}$ and $w_{3}$. The fact that the irreducible $w$ is replaced by reducible fiber function is responsible for the new cycles that will constitute the singular fiber $I_{5}$.

Let us analyze the fiber structure after the first blow-up. In the patch $\mathscr{U}_{1}$, the fiber is obtained by cutting along $w_{1} x_{1}=0$. The component $w_{1}=0$ gives the proper transform of the original fiber $C_{0}$ while $x_{1}=0$ gives a new reducible component which is the union of two rational curves $C_{1+}$ and $C_{1-}$; each intersecting $C_{0}$ at one point. These two components are disjoint in the patches $\mathscr{U}_{1}$ and $\mathscr{U}_{2}$. The proper transform of the Weierstrass equation $\mathscr{E}$ is smooth in the patches $\mathscr{U}_{1}$ and $\mathscr{U}_{2}$. In the patch $\mathscr{U}_{3}$, we do not see $C_{0}$, but we can see both $C_{1+}$ and $C_{1-}$ and their intersection point $w_{3}=x_{3}=y_{3}=0$, which is the singular locus of the proper transform of $\mathscr{E}$.

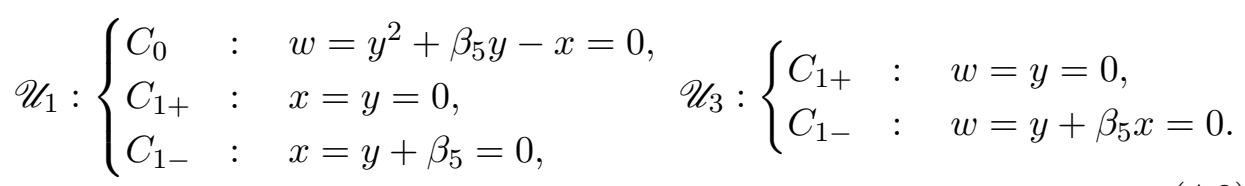

To completely smooth the variety up to codimension-two (codimension-one in the base), we have to perform an additional blow-up in the patch $\mathscr{U}_{3}$ at the $x_{3}=y_{3}=w_{3}=0$. We introduce an additional $\mathbb{P}^{2}$ with projective coordinates $\left[U_{3,1}, U_{3,2}, U_{3,3}\right]$ and the relations

$$
U_{3,2} x_{3}-U_{3,1} y_{3}=0, \quad U_{3,2} w_{3}-U_{3,3} y_{3}=0, \quad U_{3,1} w_{3}-U_{3,3} x_{3}=0 .
$$


Table 6: Defining equations for the blow-ups generating the fiber $I_{5}$.

\begin{tabular}{lccc}
\hline Patch & & Proper transform of the defining equation $\mathscr{E}$ & Fibers \\
\hline $\mathscr{U}_{1}$ & $\mathscr{E}:$ & $y^{2}+w^{2} \beta_{3} x y+\beta_{5} y=x+w^{5} x^{3} \beta_{0}+w^{3} \beta_{2} x^{2}+w \beta_{4} x$ & $x w=0$ \\
$\mathscr{U}_{3}$ & $\mathscr{E}:$ & $y^{2}+\beta_{5} x y+w \beta_{3} y=w x^{3}+w^{3} \beta_{0}+w^{2} \beta_{2} x+w \beta_{4} x^{2}$ & $w=0$ \\
$\mathscr{U}_{3,1}$ & $\mathscr{E}:$ & $y^{2}+\beta_{5} y+w \beta_{3} y=w x^{2}+w^{3} \beta_{0} x+w^{2} \beta_{2} x+w \beta_{4} x$ & $x w=0$ \\
$\mathscr{U}_{3,3}$ & $\mathscr{E}:$ & $y^{2}+\beta_{5} x y+\beta_{3} y=w^{2} x^{3}+w \beta_{0}+w \beta_{2} x+w \beta_{4} x^{2}$ & $w=0$ \\
\hline
\end{tabular}

Defining $x_{3,1}=x_{3}, y_{3,1}=U_{3,2} / U_{3,1}, w_{3,1}=U_{3,3} / U_{3,1}$, etc., the blow-up of $x_{3}=y_{3}=w_{3}=0$ is defined by the following three morphisms:

$$
\begin{array}{ll}
\varphi_{3,1}: & \left(x_{3}, y_{3}, w_{3}\right) \mapsto\left[x_{3,1}, x_{3,1} y_{3,1}, x_{3,1} w_{3,1}\right] \\
\varphi_{3,2}: & \left(x_{3}, y_{3}, w_{3}\right) \mapsto\left[y_{3,2} x_{3,2}, y_{3,2}, y_{3,2} w_{3,2}\right] \\
\varphi_{3,3}: & \left(x_{3}, y_{3}, w_{3}\right) \mapsto\left[w_{3,3} x_{3,3}, w_{3,3} y_{3,3}, w_{3,3}\right]
\end{array}
$$

where $\varphi_{3, k}$ is defined in the patch $\mathscr{U}_{3, k}: U_{3, k} \neq 0(k=1,2,3)$. The special fiber in the patch $\mathscr{U}_{3,1}$ is given by the fiber function $w_{31} x_{31}=0$ which gives two reducible components $C_{1}$ and $C_{2}$ that are composed of the rational curves $C_{1 \pm}$ and $C_{2 \pm}$. Altogether $C_{0}, C_{1 \pm}, C_{2 \pm}$ define the fiber $I_{5}$. Altogether all the components of the fiber $I_{5}$ can be seen in the patch $\mathscr{U}_{1}$ (where they can see $C_{0}$, the only component touching the section) and in the $\mathscr{U}_{3,1}$, where we have all the other components:

$$
\mathscr{U}_{3,1}: \begin{cases}C_{1+} & : \quad w=y=0 \\ C_{1-} & : \quad w=y+\beta_{5}=0, \\ C_{2+}: & x=y=0 \\ C_{2-}: & x=y+\beta_{5}+w \beta_{3}=0 .\end{cases}
$$

After this blow-up, the variety is smooth in codimension-one. As we will see next, there are singularities above loci in higher codimensions in the base. But they are all visible in the patch $\mathscr{U}_{3,1}: U_{3,1} \neq 0$ where the components $C_{1 \pm}$ and $C_{2 \pm}$ are visible. The patch $\mathscr{U}_{3,1}$ will play a central role in the rest of this paper since this is the ground where all the additional enhancements will take place. The two blow-ups yielding the fiber $I_{5}$ are summarized $^{6}$ in equations (4.8) and (4.12) and in tables 6 and 7.

\footnotetext{
${ }^{6}$ To simplify notations, we do not put the indexes and we work just with $(x, y, w)$. This is usually enough when we specify explicitly in which patch we are working.
} 
Table 7: Atlas of the blow up of the $I_{5}$ singularity. Each patch is indicated by its name and we name the components visible from that patch. In case the variety is still singular and need an additional blow-up in a given patch we indicate explicitly the singular locus.

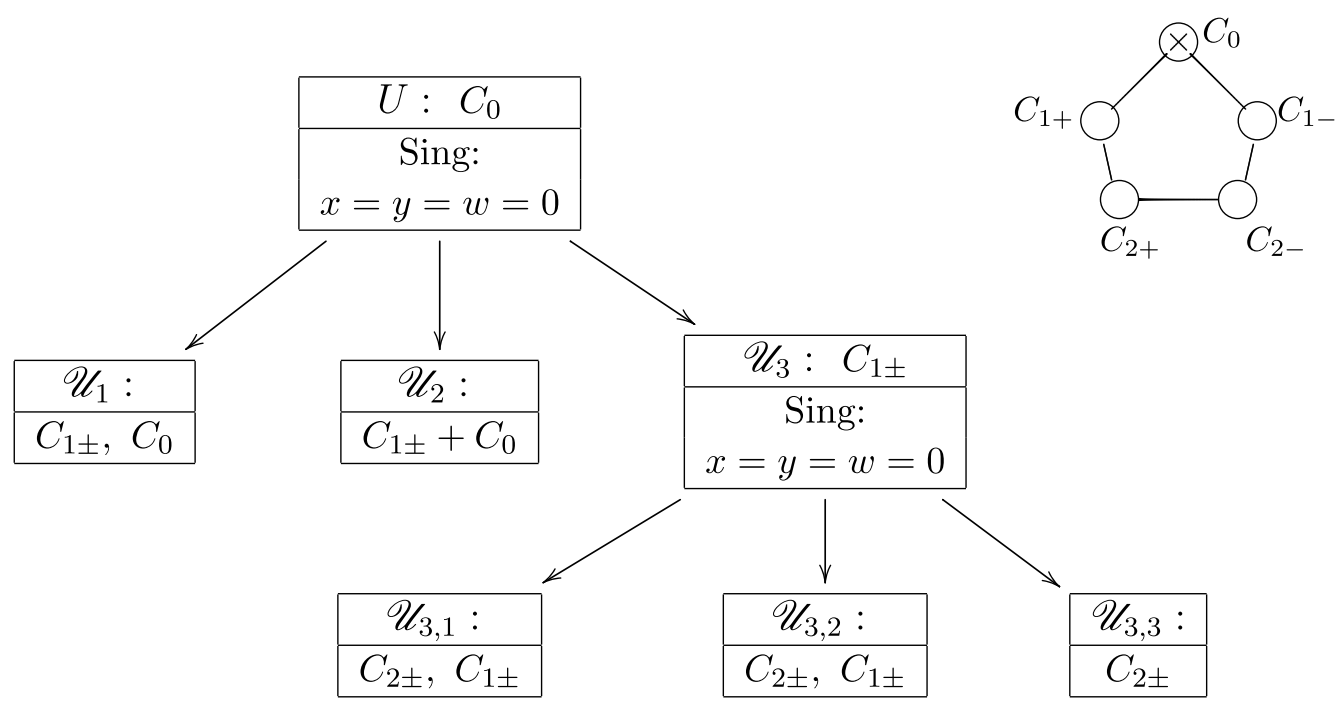

\subsection{Fiber mutation over higher codimension singularities}

After resolving the singularity above $D_{\mathfrak{s u}(5)}$, there are still extra singularities left in higher codimension in the base. With the exception of $C_{0}$, all the components of the $I_{5}$ fiber are visible in the patch $\mathscr{U}_{31}$. Since there are no singularities on $C_{0}$ and we will see that all the singularities are visible in the patch $\mathscr{U}_{31}$ :

$$
\mathscr{E} \text { in } \mathscr{U}_{31}: \quad y^{2}+\beta_{5} y+w \beta_{3} y=w x^{2}+w^{3} \beta_{0} x+w^{2} \beta_{2} x+w \beta_{4} x .
$$

In order to understand the higher codimension enhancement of the fiber, it is necessary to first analyze the extra singularities present in higher codimension. We have summarized the singular structure in table 8. We have recovered all the singular loci expected from the analysis of the discriminant with the exception of the points $\Pi_{7}$. We will see later that there is actually no enhancement of the fiber above these points.

The $I_{5}$ fiber changes dramatically over the loci in the base corresponding to higher dimensional singularities. For example, over $\Sigma_{10}: \beta_{5}=0$ and the components $C_{1+}$ and $C_{1-}$ coincide and form a unique component $C_{1}$ which intersects $C_{2+}$ and $C_{2-}$ at a common point $L_{t}$. There is another singularity $L_{x}$ sitting on $C_{1}$ away from $C_{2 \pm}$ (as long as $\beta_{4} \neq 0$ ). Over 
Table 8: Singular loci in higher codimensions. The first column indicates the singular loci, the second column expressed the location of the singularity in the base and mention the codimension. Finally the third column indicate which components of the $I_{5}$ contain the singularity. We denote by $C_{1}$ (resp. $C_{2}$ ) the node obtain when $C_{1 \pm}$ (resp. $C_{2 \pm}$ ) coincide.

\begin{tabular}{|c|c|c|c|}
\hline Sing. Locus of $\mathscr{E}$ in $\mathscr{U}_{3,1}$ & & Codim in the base of $\mathscr{E}$ & $\begin{array}{c}\text { Located on the } \\
\text { components }\end{array}$ \\
\hline$\overline{L_{t}:} x=y=w=\beta_{5}=0$ & 2 & $\left(\Sigma_{10}: \beta_{5}=0\right.$ in $\left.D_{\mathfrak{s u}(5)}\right)$ & $C_{1} \cap C_{2+} \cap C_{2-}$ \\
\hline$L_{x}: x+\beta_{4}=y=w=\beta_{5}=0$ & 2 & $\left(\Sigma_{10}: \beta_{5}=0\right.$ in $\left.D_{\mathfrak{s u}(5)}\right)$ & $C_{1}$ \\
\hline$L_{w}\left\{\begin{array}{l}y=x=w \beta_{3}+\beta_{5}=0 \\
w^{2} \beta_{0}+w \beta_{2}+\beta_{4}=0\end{array}\right.$ & 2 & $\left(\Sigma_{5}:=P=0\right.$ in $\left.D_{\mathfrak{s u}(5)}\right)$ & $C_{2+} \cap C_{2-}$ \\
\hline$p_{3}\left\{\begin{array}{l}x=y=\beta_{5}=\beta_{3}=0 \\
w^{2} \beta_{0}+w \beta_{2}+\beta_{4}=0\end{array}\right.$ & 3 & $\left(\Pi_{3}: \beta_{5}=\beta_{3}=0\right.$ in $\left.D_{\mathfrak{s u}(5)}\right)$ & $C_{2}$ \\
\hline$p_{4}: x=y=w=\beta_{5}=\beta_{4}=0$ & 3 & $\left(\Pi_{4}: \beta_{5}=\beta_{4}=0\right.$ in $\left.D_{\mathfrak{s u}(5)}\right)$ & $C_{1} \cap C_{2}$ \\
\hline
\end{tabular}

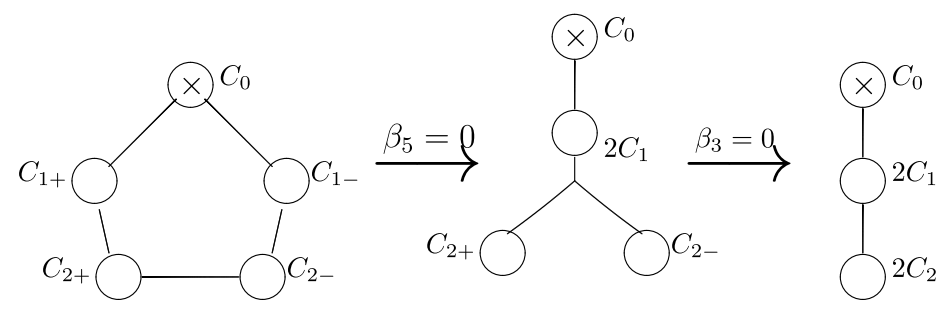

Figure 7: Higher codimension specialization of the $I_{5}$ singularity. As we go to higher codimension, there are singularities over which the $I_{5}$ fiber changes its shape due to component superposition.

$\Pi_{3}: \beta_{5}=\beta_{3}=0, C_{2+}$ and $C_{2-}$ also coincide with each other. The mutation associated with the specialization $\beta_{5}=0$ and $\beta_{5}=\beta_{3}=0$ is illustrated in figure 7. Over $\Sigma_{5}: P=0$, the fiber keeps its $I_{5}$ structure but it develops a singularity at $L_{w}$ corresponding to the intersection of $C_{2+} \cap C_{2-}$ over the locus $\Sigma_{5}$.

\section{The binomial geometry of SU(5) GUTs}

In this section, we introduce the binomial geometry that naturally appears after resolving the singularities of the SU(5) in codimension-one. We will study carefully the singularities of the binomial variety and their 
desingularization by explicit resolutions. We will do it both using the natural toric description of the binomial variety and a direct algebraic description. The binomial variety we are dealing can be seen as a higher dimension generalization of the conifold. We will review therefore the small resolution of a conifold and Atiyah's flop in some details. For a conifold singularity, there are two small resolutions connected by a flop. For the binomial variety we consider, we will have six small resolutions related by a networks of flop transitions forming a dihedral group of order 12 . We will use this analysis to fully resolve the $\mathrm{SU}(5)$ model and describe its fiber in every codimension.

In order to resolve the higher codimension singularity of the elliptic fibration, we will work in the patch $\mathscr{U}_{3,1}$ where all these singularities are visible. After introducing the variables $s$ and $t$ defined by

$$
s=y+\beta_{5}+w \beta_{3}, \quad t=x+\beta_{4}+w^{2} \beta_{0}+w \beta_{2},
$$

we see that the fibration $\mathscr{E}$ has the structure of an affine binomial variety:

$$
\mathscr{E} \text { in } \mathscr{U}_{3,1}: \quad y s-w x t=0, \quad x, w, t, y, s \in \mathbb{C} .
$$

An affine binomial variety is the vanishing locus of a binomial polynomial (the sum of two monomials). In this section we will spend some time understanding this geometry. Binomial ideals are reviewed for example in the lectures of Bernard Teissier [33]. Binomial varieties have the nice property of being affine toric varieties. Their singularities are considered to be the simplest class of non-degenerate singularities. There are several binomial varieties that naturally occur in string theory (table 9$)$. A cusp $\left(y^{2}=x^{3}\right)$ is an example of a dimension-one binomial variety, a surface with a $A_{k}$ singularity $\left(x y=z^{k+1}\right)$ is also a binomial variety. The conifold $(x y-w z=0)$ is probably the most famous binomial variety in string theory. A pinch point singularity is described by The Whitney's umbrella $\left(x^{2}=z y^{2}\right)$ and is a binomial variety that appears naturally when we take the orientifold limit of F-theory $[26,27]$. The binomial variety that we are interested in $(x w t-y s=0)$ is a beautiful example of higher dimensional singular variety. Its singular locus is composed of a bouquet of three double lines and the singularity enhances at the origin of the bouquet. In other words, we have three conifold lines all intersecting at one point where the singularity worsens. Its intersection with different linear spaces reproduce the Whitney umbrella, the cusp or the $A_{2}$ singularity. We will review the small resolution of the conifold in the next subsection as a warmup before attacking the resolution of the higher codimension singularities of $\mathscr{E}$. 
Table 9: Examples of common binomial varieties.

\begin{tabular}{lcc}
\hline Name & Defining equation & Dimension \\
\hline A curve with a cusp & $x^{2}-y^{3}=0$ & 1 \\
A surface with a $A_{k}$-point & $x y-z^{k+1}=0$ & 2 \\
The Whitney's umbrella & $y^{2}-z w^{2}=0$ & 2 \\
The conifold & $x y=z w$ & 3 \\
\hline
\end{tabular}

\subsection{Atiyah's flop and the small resolution of the conifold}

A conifold is the double point singularity of the tip of an affine quadric cone:

$$
\mathscr{C}: u_{1} u_{2}-v_{1} v_{2}=0, \quad u_{i}, v_{i} \in \mathbb{C} .
$$

The quadric cone is smooth in codimension-one and codimension-two but admits a double point singularity at the origin. It can be smoothed by a small resolution or by a blow-up. The blow-up $\pi: \hat{\mathscr{C}} \rightarrow \mathscr{C}$ of the origin resolves the singularity by replacing the origin by a ruled surface $\mathbb{F}_{0}=\mathbb{C P}^{1} \times$ $\mathbb{C P}^{1}$ defined by a quadric equation in $\mathbb{C P}^{3}$. This ruled surface $\mathbb{F}_{0}$ can be contracted to a rational curve $\mathbb{C P}^{1}$ in two different ways corresponding to the two different rulings of $\mathbb{F}_{0}$. Each of these two contractions $\bar{\pi}_{i}: \hat{\mathscr{C}} \rightarrow \hat{\mathscr{C}}_{i}$ $(i=1,2)$ defines a small resolution of the original quadric cone. These two small resolutions are related to each other by a flop transition $\hat{\mathscr{C}}_{1} \leftrightarrow-\cdots$ $\hat{\mathscr{C}}_{2}$. Alternatively $\hat{\mathscr{C}}_{i}$ can be obtained by blowing up the (non-Cartier) Weil divisor $u_{1}=v_{1}=0$ or $u_{1}=v_{2}=0$ :

$$
\hat{\mathscr{C}}_{1}:\left\{\begin{array}{l}
u_{1} \alpha-\sigma v_{1}=0, \\
v_{2} \alpha-\sigma u_{2}=0,
\end{array} \quad \hat{\mathscr{C}}_{2}:\left\{\begin{array}{l}
u_{1} \alpha-\sigma v_{2}=0, \\
v_{1} \alpha-\sigma u_{2}=0,
\end{array}\right.\right.
$$

where $u_{i}, v_{j} \in \mathbb{C}$ and $[\alpha: \sigma]$ are the projective coordinates of a $\mathbb{C P}^{1}$. The flop transition of the small resolution of the conifold was described by Atiyah in 1958 [34] (figure 8).

\subsection{Toric description of the binomial variety}

A binomial variety is always toric. $\mathscr{E}_{\mathrm{bin}}: u_{1} u_{2} u_{3}-v_{1} v_{2}=0$ is an affine toric variety as it can be seen by describing it as the variety defined by the semigroup $M$ generated by the four-vectors $m_{u_{1}}=(1,0,0,0), m_{u_{2}}=(0,1,0,0)$, $m_{u_{3}}=(0,0,1,0), m_{v_{1}}=(0,0,0,1)$ and $m_{v_{2}}=(1,1,1,-1)$ since they satisfy the relation $m_{u_{1}}+m_{u_{2}}+m_{u_{3}}=m_{v_{1}}+m_{v_{2}}$. We can also describe its algebraic torus explicitly: $\mathscr{E}_{*}=\left\{\left(u_{1}, u_{2}, u_{3}, v_{1}, v_{2}\right) \in \mathscr{E}_{\text {bin }}: u_{1} u_{2} u_{3} v_{1} v_{2} \neq 0\right)$. It is 


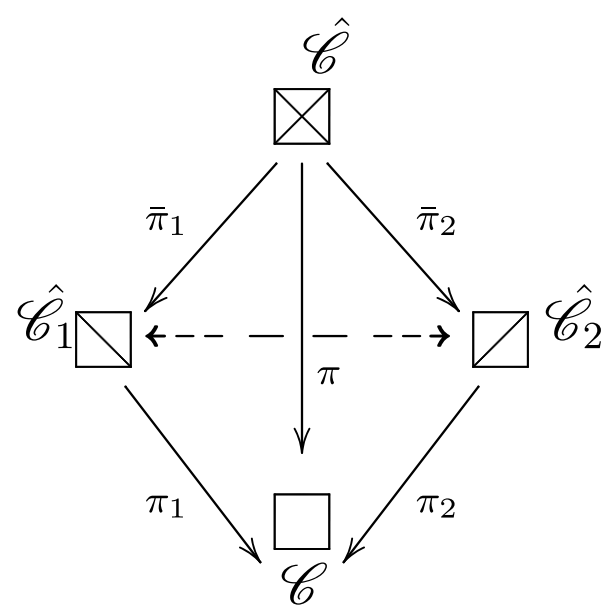

Figure 8: Flop transition $\hat{\mathscr{C}}_{1} \leftrightarrow--\rightarrow \hat{\mathscr{C}}_{2}$ between the two small resolutions of the conifold.

a four-torus as can be seen by the isomorphism:

$$
\mathbb{T}^{4}:=\left(\mathbb{C}^{*}\right)^{4} \rightarrow \mathscr{E}_{*}: \quad\left(u_{1}, u_{2}, u_{3}, v_{1}\right) \mapsto\left(u_{1}, u_{2}, u_{3}, v_{1}, u_{1} u_{2} u_{3} v_{1}^{-1}\right) .
$$

Every lattice point $m=(a, b, c, d) \in \mathbb{Z}^{4}$ defines a one-parameter subgroup as follows:

$$
\mathbb{C}^{*} \rightarrow \mathscr{E}_{*}: \lambda \mapsto \lambda^{m}=\left(\lambda^{a}, \lambda^{b}, \lambda^{c}, \lambda^{d}, \lambda^{a+b+c-d}\right) .
$$

These one-parameter subgroups form a lattice equivalent to the semi-group $M$ defined above. The dual lattice $N$ of $M$ defines the fan of the toric variety. It can alternatively be defined as the set of conditions for which the one-parameter subgroups are well defined on $\mathscr{E}_{\text {bin }}$. That is, when the following inequalities hold:

$$
a \geq 0, \quad b \geq 0, \quad c \geq 0, \quad d \geq 0, \quad a+b+c-d \geq 0
$$

These inequalities define a cone $\sigma^{\vee}$ in $\mathbb{R}^{4}$ with apex at the origin. The cone $\sigma^{\vee}$ is generated by the rows of the following matrix:

$$
\begin{aligned}
& \bar{a} \\
& \bar{b} \\
& \bar{c} \\
& c \\
& b \\
& b \\
& a
\end{aligned}\left(\begin{array}{llll}
1 & 0 & 0 & 0 \\
0 & 1 & 0 & 0 \\
0 & 0 & 1 & 0 \\
1 & 0 & 0 & 1 \\
0 & 1 & 0 & 1 \\
0 & 0 & 1 & 1
\end{array}\right)
$$

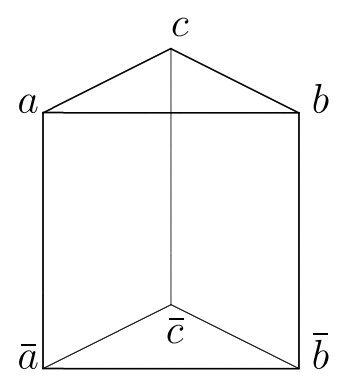


This matrix above has a very simple geometric interpretation: the rows correspond to the vertices of a triangular prism inside the three-dimensional hyper-plane

$$
\left(x_{0}, x_{1}, x_{2}, x_{3}\right) \in \mathbb{R}^{4}: x_{0}+x_{1}+x_{2}=1 .
$$

The cone is completely specified by the prism. We can visualize the cone by drawing the prism in a three-dimensional space simply by discarding the first column. By doing so, we have to keep in mind that a point $\left(x_{1}, x_{2}, x_{3}\right) \in$ $\mathbb{Z}^{3}$ in the three-dimensional drawing space corresponds to an actual point of the prism if we can find an $x_{0} \in \mathbb{Z}$ such that $x_{0}+x_{1}+x_{2}=1$. The different faces of the cone are also in one-to-one relation with the invariant loci of the torus action. A face of dimension $d$ in the prism corresponds to a face of dimension $(d+1)$ of the cone $\sigma^{\vee}$ and therefore to a torus of dimension $4-(d+1)=3-d$. In particular, the prism itself corresponds to an invariant point of the torus action, a facet of the prism corresponds to an invariant curve, an edge of the prism corresponds to an invariant surface of the torus action and a vertex corresponds to a toric divisor.

\subsection{Small resolutions and network of flop transitions}

The singular locus of the variety $\mathscr{E}_{\text {bin }}$ is a bouquet of three lines of conifold singularities enhancing at the center of the bouquet where the three lines meet at one point. In the toric description, the dual cone of the variety $\mathscr{E}_{\text {bin }}$ is determined by a triangular prism as explained above. The three conifold lines of $\mathscr{E}_{\text {bin }}$ correspond to the three rectangular facets of the prism while the center of the bouquet is the interior of the prism. A resolution of all the singularities of $\mathscr{E}_{\mathrm{bin}}$ is obtained by a simplicial refinement of the prism with cells of unit lattice volume. In particular, a small resolution occurs when the refinement does not add any new vertex (figure 9). This is because a vertex corresponds to an invariant divisor and by definition a small resolution does not have any divisor in its exceptional locus. It is possible to get a simplicial subdivision of any rational polytope by performing a succession of starsubdivisions of its fan. A star-subdivision is defined by a choice of a point $v$ and the following algorithm: any cone that contains $v$ is replaced by the joins of its faces with the ray through $v$; each cone not containing $v$ is left unchanged. A star subdivision defines a proper birational map since after the subdivision, the fan still has the same support. If the center of the star subdivision is already a point of the polytope, the birational map is small since there are no new vertices created.

A small resolution of $\mathscr{E}$ bin is given by the classical subdivision of the prism into three tetrahedra. Such a subdivision can always be expressed as two 


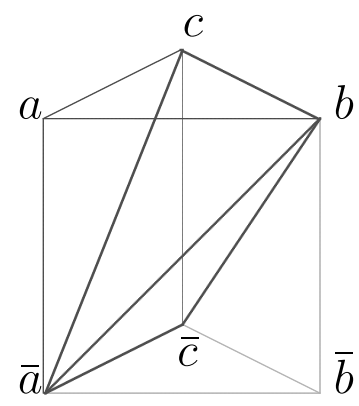

Figure 9: The small resolution $T(\bar{a}, b)$ of the binomial variety $y s-w x t=0$ is obtained by a refinement of the triangular prism $(a, b, c, \bar{a}, \bar{b}, \bar{c})$ into a sum of three tetrahedra, namely $(\bar{a}, a, b, c),(\bar{a}, \bar{c}, b, c)$ and $(\bar{a}, \bar{b}, \bar{c}, b)$.

star-subdivisions with centers $v_{1}$ and $v_{2}$ where the two centers are the end points of the diagonal of one of the three rectangular facets of the prism. We will denote such a resolution $T\left(v_{1}, v_{2}\right)$. Let us consider as an example the resolution $T(\bar{a}, b)$ defined by the vertex $\bar{a}$ and $b$. This is illustrated in figure 10. The star-subdivision determines by the vertex $\bar{a}$ generates a subdivision of the prism into a tetrahedron $(\bar{a}, a, b, c)$ and the rectangular pyramid $(\bar{a}, \bar{c}, \bar{b}, b, c)$ as illustrated in the first two pictures on the left of figure 10. The second star-subdivision is determined by the vertex $b$. It does not modify the tetrahedron $(\bar{a}, a, b, c)$ but it subdivides the rectangular pyramid into two tetrahedra $(\bar{a}, \bar{c}, b, c)$ and $(\bar{a}, \bar{b}, b)$. Altogether, the small resolution $T(\bar{a}, b)$ defines the following subdivision of the prism into three tetrahedra as illustrated in figure 10:

$$
T(\bar{a}, b)=\{(\bar{a}, a, b, c), \quad(\bar{a}, \bar{c}, b, c), \quad(\bar{a}, \bar{b}, \bar{c}, b)\} .
$$

We could have also considered the small resolution

$$
T(\bar{a}, c)=\{(\bar{a}, a, b, c),(\bar{a}, b, c, \bar{b}),(\bar{a}, \bar{b}, \bar{c}, c)\} .
$$

The first star-subdivision with center $\bar{a}$ gives the same splitting into a tetrahedron and a rectangular pyramid as in $T(\bar{a}, b)$. But the second starsubdivision uses the other possible splitting of the pyramid into two tetrahedrons. The difference between the resolutions $T(\bar{a}, b)$ and $T(\bar{a}, c)$ is then a choice on how to split the rectangular pyramid into two tetrahedra. This is exactly the same difference between the two small resolutions of the conifold. More generally, two resolutions $T(\bar{u}, v)$ and $T(\bar{p}, q)$ are related by a (conifold) flop transition defined by switching the choice of a diagonal plane if and only if $u=p$ or $v=q$. Altogether, we have a total of six small resolutions 

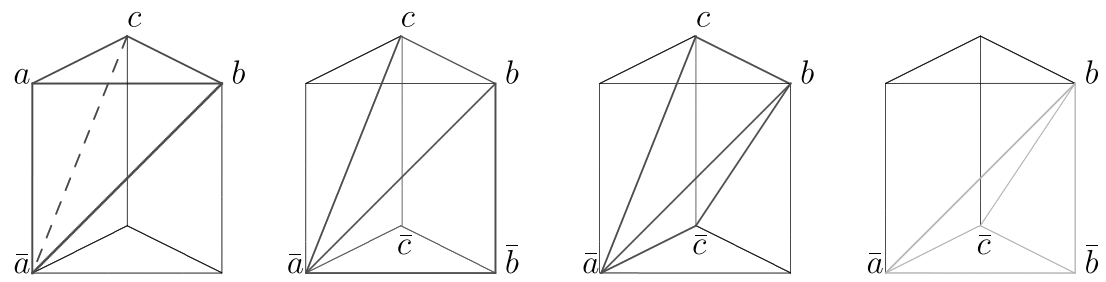

Figure 10: The resolution $T(\bar{a}, b)$. We split the prism into a tetrahedron $(\bar{a}, a, b, c)$ and a rectangular pyramid $(\bar{a}, b, c, \bar{b}, \bar{c})$ using the plane $(\bar{a}, b, c)$. We then split the rectangular pyramid into the two tetrahedra $(\bar{a}, \bar{c}, c, b)$ and $(\bar{a}, \bar{b}, \bar{c}, b)$ using the plane $(\bar{a}, \bar{c}, b)$.

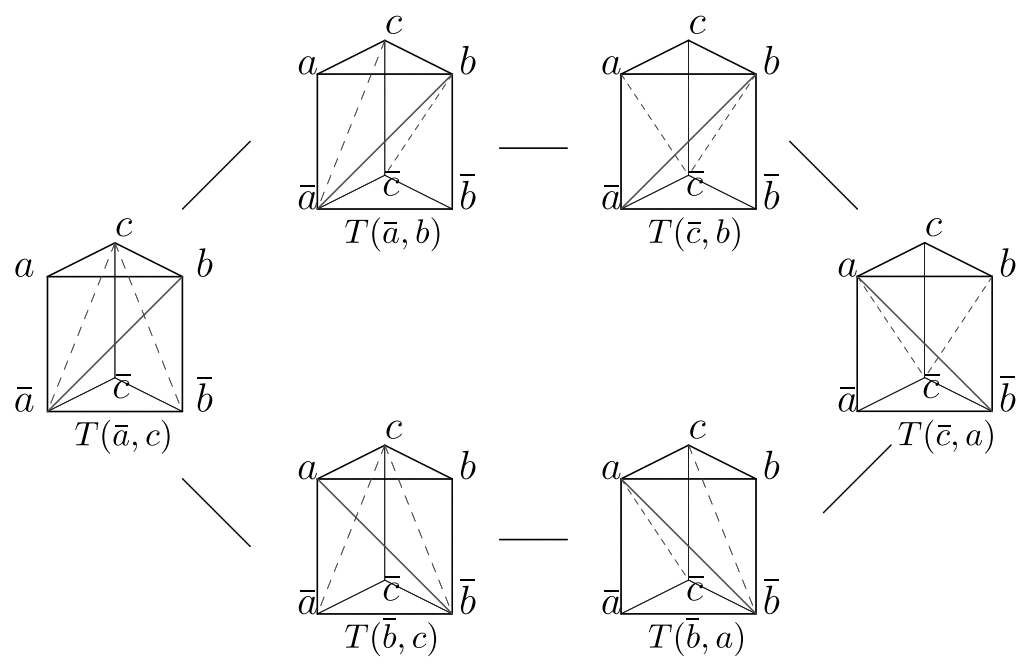

Figure 11: The six small resolutions of the binomial variety ys $-w x t=0$. Each of the small resolution is obtained by a subdivision of the prism into three tetrahedra. All the possible choices are connected by a sequence of such flop transitions on which the symmetric group $\mathscr{S}_{3}$ acts transitively.

as represented in figure 11:

$$
T(\bar{a}, b), \quad T(\bar{a}, c), \quad T(\bar{b}, c), \quad T(\bar{b}, a), \quad T(\bar{c}, a), \quad T(\bar{c}, b) .
$$

The group $\mathscr{S}_{3}$ of permutations of three letters $\{a, b, c\}$ acts transitively on this set of resolutions. They are connected to each other by a network of flop transitions as represented in figure 11. The exceptional locus over a given cone is the union of all the minimal cones intersecting its relative interior. For the six resolutions we obtained, each rectangular facet admits as its exceptional locus a $\mathbb{C P}^{1}$-bundle over the singular lines and the $\mathbb{C P}^{1}$ fiber 


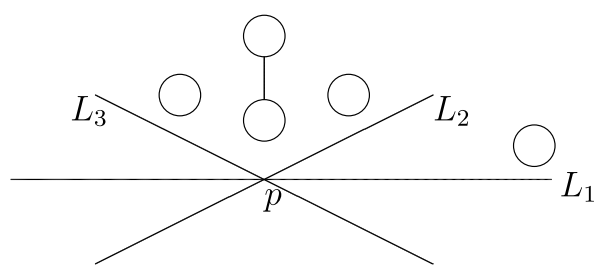

Figure 12: The exceptional locus of the small resolution of the binomial variety $u_{1} u_{2} u_{3}=v_{1} v_{2}$ is a $\mathbb{C P}^{1}$-bundle with a fiber which enhances at the origin to two $\mathbb{C P}^{1}$ intersecting at a point.

enhances to two intersecting $\mathbb{C P}^{1}$ at the common intersection of the three lines. The structure of the exceptional locus is illustrated in figure 12 .

\subsection{Algebraic description of the network of small resolutions}

The toric description shows that a small resolution can be obtained by blowing-up two Weil divisors corresponding to two vertices that are opposite ends of the same diagonal of a given rectangular facet of the prism. This can be easily implemented algebraically. We will derive it slightly differently using an analogy with the small resolution of the conifold. Consider the defining equation of the binomial variety

$$
u_{1} u_{2} u_{3}=v_{1} v_{2}, \quad\left(u_{1}, u_{2}, u_{3}, v_{1}, v_{2}\right) \in \mathbb{C}^{5} .
$$

Assuming for a moment that $v_{1} v_{2} \neq 0$, we can rewrite it as the following fractional relation:

$$
\frac{u_{1}}{v_{1}} \frac{u_{2}}{v_{2}} u_{3}=1
$$

We then introduce two projective lines $\mathbb{P}^{1}$ with projective coordinates $\left[\alpha_{ \pm}, \sigma_{ \pm}\right]$and we identify their affine coordinates with $u_{1} / v_{1}$ and $u_{2} / v_{2}$ :

$$
\frac{\sigma_{+}}{\alpha_{+}}=\frac{u_{1}}{v_{1}}, \quad \frac{\sigma_{-}}{\alpha_{-}}=\frac{u_{2}}{v_{2}} .
$$

Using the defining equation of the binomial variety, we get the small resolution

$$
\begin{aligned}
\mathscr{B}_{12}: & \left\{\begin{array}{l}
u_{1} \alpha_{+}-\sigma_{+} v_{1}=0 \\
u_{2} \alpha_{-}-\sigma_{-} v_{2}=0 \\
\alpha_{+} \alpha_{-}-\sigma_{+} \sigma_{-} u_{3}=0 .
\end{array}\right. \\
& \text { where }\left[\alpha_{+}: \sigma_{+}\right] \times\left[\alpha_{-}: \sigma_{-}\right] \in \mathbb{F}_{0}=\mathbb{C P}^{1} \times \mathbb{C P}^{1} .
\end{aligned}
$$


Since the Jacobian has maximal rank, this is a smooth variety. The exceptional locus over a general point of $L_{1} \cup L_{2} \cup L_{3}$ is just a $\mathbb{C P}^{1}$ inside $\mathbb{F}_{0}=$ $\mathbb{C P}^{1} \times \mathbb{C P}^{1}$. More precisely, $L_{1}$ gives a $\mathbb{C P}^{1}$ that represents a ruling of $\mathbb{F}_{0}$, while $L_{2}$ gives a $\mathbb{C P}^{1}$ that represents the other ruling and $L_{3}$ is given by a diagonal $\mathbb{C P}^{1}$ which is not a ruling of $\mathbb{F}_{0}$ but the conic $\alpha_{+} \alpha_{-}-\sigma_{+} \sigma_{-} u_{3}=0$ in $\mathbb{F}_{0}$. At the point $p=L_{1} \cap L_{2}=L_{2} \cap L_{3}=L_{1} \cap L_{3}=L_{1} \cap L_{2} \cap L_{3}$, where the three lines meet, the exceptional locus enhances to two $\mathbb{C P}^{1}$ intersecting transversely. Using this algebraic description, we can also see that there are six different small resolutions. Indeed, the small resolution $\mathscr{B}_{12}$ can be replaced by a different "flop dual" resolution defined by permuting $\left(u_{1}, u_{2}, u_{3}\right)$. In total there are six possibilities. If we denote by $(i, j, k)$ a permutation of $(1,2,3)$, we define the resolution $\pi_{i j}: \mathscr{B}_{i j} \rightarrow \mathscr{B}$ as follows:

$$
\begin{aligned}
\mathscr{B}_{i j}: & \left\{\begin{array}{l}
u_{i} \alpha_{+}-\sigma_{+} v_{1}=0, \\
u_{j} \alpha_{-}-\sigma_{-} v_{2}=0, \\
\alpha_{+} \alpha_{-}-\sigma_{+} \sigma_{-} u_{k}=0 .
\end{array}\right. \\
& \text { where }\left[\alpha_{+}: \sigma_{+}\right] \times\left[\alpha_{-}: \sigma_{-}\right] \in \mathbb{F}_{0}=\mathbb{C P}^{1} \times \mathbb{C P}^{1} .
\end{aligned}
$$

The resolution $\pi_{i j}: \mathscr{B}_{i j} \rightarrow \mathscr{B}$ is obtained by blowing-up the two Weil divisors $u_{i}=v_{1}=0$ and $u_{j}=v_{2}=0$. Since these Weil divisors are not Cartier, they actually do not lead to an exceptional divisor, the exceptional locus being in codimension-two. These two divisors correspond to some vertexes $\bar{a}$ and $b$ of the prism and therefore the resolution $\pi_{i j}$ is the same as the toric resolution $T(\bar{a}, b)$. The flop transition between the resolutions $\mathscr{B}_{i j}$ and $\mathscr{B}_{i k}$ can be understood as a geometric procedure under which the $\mathbb{C P}^{1}$ fiber of the exceptional locus coming from the blow-up of $u_{j}=v_{2}=0$ is blown-down and replaced by the $\mathbb{C P}^{1}$ coming from the blowup of $u_{k}=v_{2}=0$. We can give a similar description for the flop transition between $\mathscr{B}_{i j}$ and $\mathscr{B}_{k j}$. Any resolution $\mathscr{B}_{i j}$ can be mapped to any other $\mathscr{B}_{i^{\prime} j^{\prime}}$ by a succession of such flop transitions as described by the loop (compare with figure 11):

$$
(12)-(13)-(23)-(21)-(31)-(32)-(12) \text {. }
$$

\section{Small resolutions for SU(5) GUTs}

The small resolutions $\pi_{i j}: \mathscr{B}_{i j} \rightarrow \mathscr{B}$ of the binomial variety $\mathscr{B}: u_{1} u_{2} u_{3}-$ $v_{1} v_{2}=0$ presented in the previous section can be pulled-back to $\mathscr{E}$ to provide small resolutions for all the higher codimension singularities of the SU(5) GUT geometry $\mathscr{E}$. This procedure can be summarized by the following 
diagram:

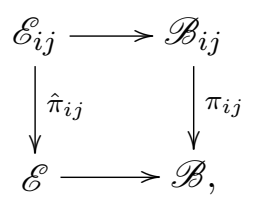

where the map $\mathscr{E} \rightarrow \mathscr{B}$ associated to each point $p$ of $\mathscr{E}$, the point $\left(u_{1}, u_{2}, u_{3}\right.$, $\left.v_{1}, v_{2}\right)$ of $\mathscr{B}$ such that

$$
u_{1}=x, \quad u_{2}=w, u_{3}=t, v_{1}=y, \quad v_{2}=s
$$

We recall that $t=x+\beta_{4}+\beta_{2} w+\beta_{0} w^{2}$ and $s=y+\beta_{5}+\beta_{3} w$. Denoting by $\left[\alpha_{ \pm}: \sigma_{ \pm}\right]$the projective coordinates of two $\mathbb{C P}^{1}$, the six small resolutions are:

$$
\begin{aligned}
& \begin{array}{l}
\mathscr{E}_{w x} \quad \begin{array}{l}
w \alpha_{+}-\sigma_{+} y=0, \\
x \alpha_{-}-\sigma_{-} s=0, \\
\alpha_{-} \alpha_{+}-\sigma_{-} \sigma_{+} t=0,
\end{array} \quad\left\{\begin{array} { l } 
{ t \alpha _ { + } - \sigma _ { + } y = 0 , } \\
{ w \alpha _ { - } - \sigma _ { - } s = 0 , } \\
{ \alpha _ { - } \alpha _ { + } - \sigma _ { - } \sigma _ { + } x = 0 , }
\end{array} \quad \left\{\begin{array}{l}
x \alpha_{+}-\sigma_{+} y=0, \\
t \alpha_{-}-\sigma_{-} s=0, \\
\alpha_{-} \alpha_{+}-\sigma_{-} \sigma_{+} w=0,
\end{array}\right.\right.
\end{array} \\
& \begin{array}{ll}
\mathscr{E}_{w t} & \mathscr{E}_{x w} \\
t \alpha_{-}-\sigma_{-} s=0, \\
\alpha_{-} \alpha_{+}-\sigma_{-} \sigma_{+} x=0,
\end{array} \quad\left\{\begin{array} { l } 
{ x \alpha _ { + } - \sigma _ { + } y = 0 , } \\
{ w \alpha _ { - } - \sigma _ { - } s = 0 , } \\
{ \alpha _ { - } \alpha _ { + } - \sigma _ { - } \sigma _ { + } t = 0 , }
\end{array} \quad \left\{\begin{array}{l}
t \alpha_{+}-\sigma_{+} y=0, \\
x \alpha_{-}-\sigma_{-} s=0, \\
\alpha_{-} \alpha_{+}-\sigma_{-} \sigma_{+} w=0 .
\end{array}\right.\right.
\end{aligned}
$$

The exceptional locus is a $\mathbb{C P}^{1}$-bundle over the bouquet $L_{x} \cup L_{w} \cup L_{t}$, but the fiber enhances to two intersecting $\mathbb{C P}^{1}$ over the center of the bouquet:

$$
\hat{\pi}_{i j}^{-1}\left(x \in L_{x} \cup L_{w} \cup L_{t}-\left\{p_{4}\right\}\right)=\bigodot^{\mathbb{C P}^{1}}, \hat{\pi}_{i j}^{-1}\left(p_{4}\right)=\bigcirc^{\mathbb{C P}^{1}}
$$

It is easy to see (for example, by computing the Jacobian) that all the six resolutions are smooth. Although the six small resolutions $\mathscr{B}_{i j}$ of the binomial variety $\mathscr{B}$ had a similar fiber structure, their uplifts $\mathscr{E}_{i j}$ to the SU(5) geometry would be significantly different from each. The dependence of $s$ and $t$ on the base through the sections $\beta_{0}, \beta_{2}, \beta_{3}, \beta_{4}$ and $\beta_{5}$ will be responsible for the enhancement of fibers of the $\mathrm{SU}(5)$ model. In the process of adding the extra nodes coming from the exceptional loci, we will get new types of fibers. 

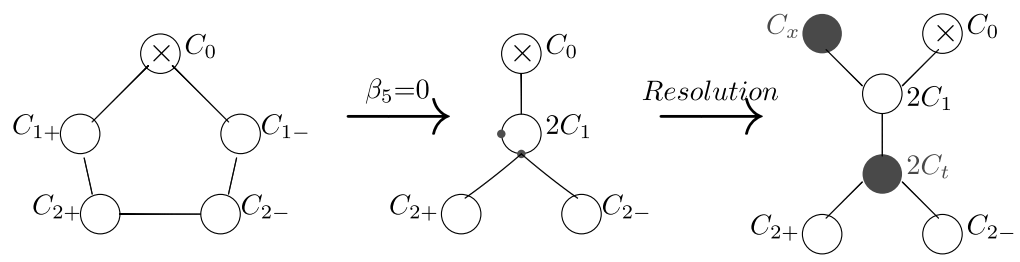

Figure 13: The codimension-two fiber enhancement $\tilde{A}_{4} \rightarrow \tilde{D}_{5}$. It corresponds to the resolution of codimension-two singularities above the curve $w=\beta_{5}=0$.

\subsection{Codimension-two singular fibers: $\tilde{A}_{5}$ and $\tilde{D}_{5}$ enhancements}

A general fiber of $\mathscr{E}$ over the divisor $D_{\mathfrak{s u}(5)}$ is a closed chain of five $\mathbb{C P}^{1}$ nodes, namely $C_{0}, C_{1 \pm}$ and $C_{2 \pm}$. In order to analyze the structure of the fiber of the resolutions $\mathscr{E}_{a b}$, we just have to understand what is happening at the singular locus $L_{t} \cup L_{x} \cup L_{w}$. We notice that $L_{t}$ and $L_{x}$ are both located above the curve $\Sigma_{10}: \beta_{5}=0$ of the base, whereas $L_{w}$ is located above $\Sigma_{5}: P=0$. The $I_{5}$ fiber undergoes a topological change over these two curves. Over the locus $\beta_{5}=0$, the curves $C_{1+}$ and $C_{1-}$ coincide to form a rational curve $C_{1}$ of multiplicity 2 . This rational curve $C_{1}$ intersects $C_{2+}$ and $C_{2-}$ at a unique point which corresponds to $L_{t}$. When $\beta_{4} \neq 0$, there is another singular point on $C_{1}$ away from $L_{t}$, this is the singularity $L_{x}$. The small resolution of $\mathscr{E}_{0}$ replaces on each fiber over $\beta_{5}=0$, the singular points $L_{t}$ and $L_{x}$ by the rational curves $C_{t}$ and $C_{x}$. The resulting fiber has the structure of an affine diagram $\tilde{D}_{5}$. This enhancement is reviewed in figure 13. Above the curve $\Sigma_{5}$, the singular point $L_{w}$ corresponds fiberwise to a singular point sitting at the intersection of $C_{2+}$ and $C_{2-}$. Any of the six small resolutions replace that point by a $\mathbb{C P}^{1}$ that we call $C_{w}$. This leads to an enhancement of the fiber $I_{5}$ to a fiber $I_{6}$ with dual graph $\tilde{A}_{5}$. This is presented in figure 14. The curves $C_{x}, C_{t}$ and $C_{w}$ are realized differently for each resolution $\mathscr{E}_{i j}{ }^{7}$.

\subsection{An affine $\tilde{D}_{6}$ enhancement in codimension-three}

When we were analyzing the structure of the fibers in codimension-two we have assumed that $\beta_{3} \beta_{4} \neq 0$ in order to obtain the fiber $I_{6}$ and that $\beta_{4} \neq 0$

\footnotetext{
${ }^{7}$ In general given a curve $C_{\ell}$ and the resolution $\mathscr{E}_{i j}$, if $\ell=i, C_{\ell}$ is defined by $L_{\ell}$ together with the condition $u_{i} \alpha_{+}=\alpha_{+} \alpha_{-}=0$, therefore it is defined by the $\mathbb{C P}^{1}: \alpha_{+}=0$, that is the line $\mathbb{C P}^{1} \times[0: 1]$ in $\mathbb{F}_{0}$. In the same way if $\ell=j, C_{\ell}$ we be given by $\alpha_{-}=0$ corresponding to the line $[0: 1] \times \mathbb{C P}^{1}$ in $\mathbb{F}_{0}$. Finally if $(\ell \neq i, j)$, then $C_{\ell}$ is defined by the quadratic equation $\alpha_{-} \alpha_{+}-\sigma_{-} \sigma_{+} u_{k}=0$ in $\mathbb{F}_{0}$. We call it a diagonal $\mathbb{C P}^{1}$ since it does not correspond to one of the ruling of $\mathbb{F}_{0}$.
} 


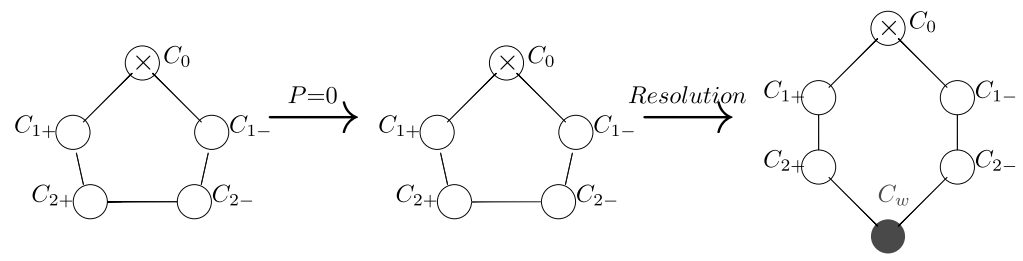

Figure 14: The codimension-two fiber transition $\tilde{A}_{4} \rightarrow \tilde{A}_{5}$ above the curve $P=0$ (assuming that $\beta_{3} \neq 0$ ). This turns the fiber $I_{5}$ into a Kodaira fiber $I_{6}$ which corresponds to the extended Dynkin diagram $\tilde{A}_{5}$.

to obtain the fiber with dual graph $\tilde{D}_{5}$. If we allow $\beta_{3}=0$ or $\beta_{4}=0$ we are in codimension-three and we can expect a enhancement of the singular fibers. The specialization $\beta_{3}=\beta_{5}=0$ and $\beta_{4}=\beta_{5}=0$ can be geometrically described as collisions of singular fibers $I_{6}$ and $\tilde{D}_{5}$ located above $P=0$ and $\beta_{5}=0$. We will first consider the case

$$
\Pi_{3}: \beta_{5}=\beta_{3}=0
$$

and we will analyze the case $\beta_{5}=\beta_{4}=0$ later. As we specialize to $\beta_{5}=$ $\beta_{3}=0$ in the SU(5) divisor, the curves $C_{1+}$ and $C_{1-}$ (resp. $C_{2+}$ and $C_{2-}$ ) coincide and determine a unique curve of multiplicity 2 that we call $C_{1}$ (resp. $C_{2}$ ). Each of our six small resolutions replaces on each fiber of $\beta_{5}=\beta_{3}=0$ the points corresponding to $L_{x}$ and $L_{t}$ by a $\mathbb{C P}^{1}$ as it was done already in codimension-two. But something new happens for the locus $L_{w}$. When we specialize to $\beta_{3}=\beta_{5}=0$, the locus $L_{w}: x=y=\beta_{5}+\beta_{3} w=\beta_{4}+$ $\beta_{2} w+\beta_{0} w^{2}=0$ reduces to $x=y=\beta_{5}=\beta_{3}=\beta_{4}+\beta_{2} w+\beta_{0} w^{2}=0$. This defines two points $L_{w \pm}$ on the component $C_{2}$ of the fiber above $\beta_{5}=\beta_{3}=0$. Indeed, the equation $\beta_{4}+\beta_{2} w+\beta_{0} w^{2}=0$ gives two different values $w_{ \pm}$for $w$ in terms of $\beta_{4}, \beta_{2}, \beta_{0}$. We can think of this as the splitting of the locus $L_{w}$ into two different loci $L_{w \pm}$ :

$$
L_{w} \stackrel{\beta_{3}=\beta_{5}=0}{\longrightarrow} L_{w \pm}, \quad w_{ \pm}=\frac{-\beta_{2} \pm \sqrt{\beta_{2}^{2}-4 \beta_{4} \beta_{0}}}{2 \beta_{0}^{2}} .
$$

Since we are in codimension-three, we can safely assume that in general $\beta_{0} \neq 0$ and $\beta_{2}^{2}-4 \beta_{4} \beta_{0} \neq 0$ so that we indeed have two values for $w$ which correspond to two disjoint points on the node $C_{2}$. In the resolution, each of these points $L_{w \pm}$ is replaced by a $\mathbb{C P}^{1}$ that we call, respectively, $C_{w+}$ and $C_{w-}{ }^{8}$ Altogether, we see that for any of our six small resolutions, over

\footnotetext{
${ }^{8}$ When $\beta_{3} \neq 0$, there was a unique value for $w$ since the equation $\beta_{5}+\beta_{3} w=0$ had a unique solution for $w$. The locus $P=0$ was obtained as a compatibility condition with the other relation $\beta_{4}+\beta_{2} w+\beta_{0} w^{2}=0$ by replacing $w$ by the solution $-\beta_{5} / \beta_{3}$.
} 

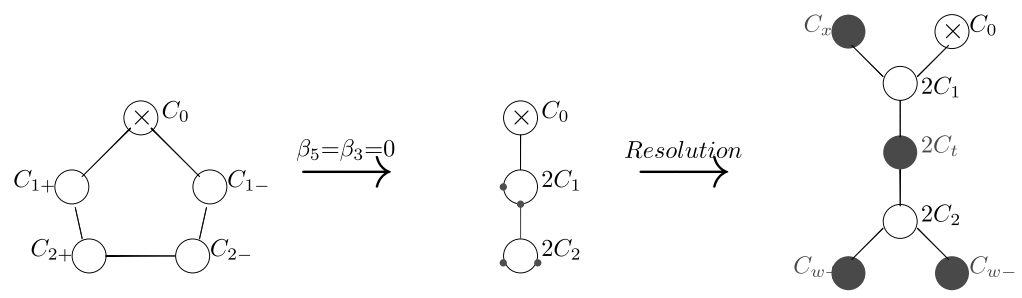

Figure 15: Fiber above $\Pi_{3}: \beta_{5}=\beta_{3}=0$ in the SU(5) divisor. After the resolution we get a fiber with dual graph an affine Dynkin diagram $\tilde{D}_{6}$.

$\beta_{5}=\beta_{3}=0$ in the $\mathrm{SU}(5)$-divisor, we get a fiber which has the structure of an affine Dynkin diagram $\tilde{D}_{6}$. The singular points $L_{w \pm}$ are replaced by $\mathbb{C P}^{1} \mathrm{~s}$ that are realized as hypersurfaces in the projective quadric $\mathbb{F}_{0}$ with the same equation as $C_{w}$ (figure 15).

\subsection{Exotic fibers in codimension-three}

We will now consider the enhancement of the singular fiber at the points

$$
\Pi_{4}: \beta_{5}=\beta_{4}=0,
$$

in the $\mathrm{SU}(5)$ divisor $D_{\mathfrak{s u}(5)}$. These are codimension-three points in the base of the elliptic fibration. They is a sublocus of the intersection of the curves $\Sigma_{5}$ and $\Sigma_{10}$. Above $\Pi_{4}$, the nodes $C_{1+}$ and $C_{1-}$ merge into a unique node $C_{1}$ of multiplicity 2. Moreover, the nodes $C_{2+}, C_{2-}$ and $C_{1}$ all intersect at the same point $p_{4}: \beta_{5}=\beta_{4}=x=y=w=0$, which is the common intersection of the three lines $L_{x}, L_{w}$ and $L_{t}$. In the toric description of the resolution of the binomial variety, the point $p_{4}$ is the interior of the prism. For any of our six small resolutions, its proper transform is the union of two ruling of $\mathbb{F}_{0}$ :

$$
\alpha_{-} \alpha_{+}=0 .
$$

This shows that $p_{4}$ is replaced by the union of two $\mathbb{P}^{1} \mathrm{~S}$ defined, respectively, by $\alpha_{-}=0$ and $\alpha_{+}=0$ inside $\mathbb{F}_{0}=\mathbb{P}^{1} \times \mathbb{P}^{1}$. We recall that we parametrize the projective cone $\mathbb{F}_{0}$ by the projective coordinates $\left[\alpha_{-}: \sigma_{-}\right]$and $\left[\alpha_{+}\right.$: $\left.\sigma_{+}\right]$. We will denote the node $x=y=w=t=s=\alpha_{-}=0$ and $x=y=w=$ $t=s=\alpha_{+}=0$, respectively, as $C_{p-}$ and $C_{p+}$. These two rational curves intersect transversally at the point $\alpha_{-}=\alpha_{+}=0=x=y=w=\beta_{5}=\beta_{4}=$ 0 . In order to understand the structure of the new fiber above the point $\beta_{5}=\beta_{4}=0$ of the SU(5) divisor, we need to clarify how the two new nodes $C_{p \pm}$ connect to the nodes $C_{1}, C_{2+}$ and $C_{2-}$. It is easy to see that in any of the six small resolutions the nodes $C_{p+}$ (resp. $C_{p-}$ ) is connected to $C_{2+}$ 


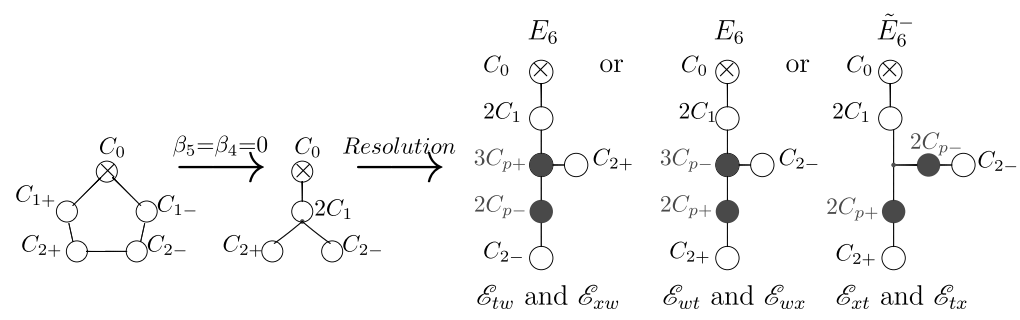

Figure 16: Codimension-three singularity in the base located at the points $\Pi_{4}: \beta_{5}=\beta_{4}=0$ of the $\mathrm{SU}(5)$-divisor. The first two fibers on the left have the structure of a projective $E_{6}$ Dynkin diagram.

(resp. $C_{2-}$ ) so that we have a chain $C_{2-}-C_{p-}-C_{p+}-C_{2+}$. In order to get the full structure of the fiber, we just need to determine how the node $C_{1}$ intersects that chain. We have the following three behaviors according the small resolution we consider: $C_{1}$ intersects $C_{p+}$ but not $C_{p-}$ (for $\mathscr{E}_{t w}$ and $\left.\mathscr{E}_{x w}\right), C_{1}$ intersects $C_{p-}$ but not $C_{p+}$ (for $\mathscr{E}_{w t}$ and $\mathscr{E}_{w x}$ ), $C_{1}$ intersects both $C_{p+}$ and $C_{p-}$ (for $\mathscr{E}_{t x}$ and $\mathscr{E}_{x t}$ ). The corresponding dual graphs are represented in figure 16 .

\subsection{Flop transitions and codimension-three singular fibers}

The different small resolutions of the binomial variety $\mathscr{E}_{\text {bin }}$ lead to six different small resolutions of the $\mathrm{SU}(5)$ geometry:

$$
\left\{\mathscr{E}_{x t}, \mathscr{E}_{w x}, \mathscr{E}_{t w}, \mathscr{E}_{w t}, \mathscr{E}_{t x}, \mathscr{E}_{x w}\right\}
$$

Each of them corresponds to a distinct resolution of the original four-fold geometry. These six four-folds have different topology and are related to each other by a network of flop transitions under which some rational curves inside codimension-two and codimension-three singular fibers shrink to a point and get replaced by other rational curves. These flop transitions do not modify the structure of the fiber above codimension-two loci. However in codimension-three in the base, the dual graph is not necessary preserved over the points $\Pi_{4}$. At these points, the rational curves $C_{p \pm}$ can change their intersection with the curve $C_{1}$ leading in this way to three different types of fibers. The fibers we get above the point $\Pi_{4}$ are not Kodaira fibers. In the case of the resolutions $\mathscr{E}_{w x}, \mathscr{E}_{t w}, \mathscr{E}_{w t}$ and $\mathscr{E}_{x w}$, the fiber is a projective $E_{6}$ Dynkin diagram that should not be confused with the affine $\tilde{E}_{6}$ which is usually conjectured to appear in the F-theory literature in order to have the 
Yukawa couplings of the up-type quarks ${ }^{9}$. For the resolution $\mathscr{E}_{x t}$ and $\mathscr{E}_{t x}$, the fiber is not even a Dynkin diagram but a bouquet composed of three twochains meeting at a common point. We have seen that the discrete group $\mathscr{S}_{3}=\mathrm{Dih}_{3}$ acts transitively on these six four-folds. It can be described by the permutation of the three elements $\{x, t, w\}$. This group acts transitively on the six small resolutions $\mathscr{E}_{a b}$. The group $\mathscr{S}_{3}$ contains two elements of order 3 and three elements of order 2 which are just transpositions. The elements of order 3 (that is $(x w t)$ or $(t w x)$ ) organize the six small resolutions into two orbits:

$$
\left\{\mathscr{E}_{x t}, \mathscr{E}_{w x}, \mathscr{E}_{t w}\right\} \quad \text { and } \quad\left\{\mathscr{E}_{w t}, \mathscr{E}_{t x}, \mathscr{E}_{x w}\right\}
$$

These two orbits are exchanged by the transpositions $(x t),(x w)$ or $(t w)$. Among all the permutations, only the transposition $(x t)$ preserves the structure of all dual graphs. Using the action of the group $\mathscr{S}_{3}$, it is convenient to organize the six small resolutions into an hexagram as represented in figure 17. The hexagram is composed of two triangles, each representing an orbit under the permutation of order three. The $\mathbb{Z}_{2}$ north-south symmetry is given by the permutation $(x t)$ which preserves all the dual graphs. The central involution which turns any $\mathscr{E}_{i j}$ into $\mathscr{E}_{j i}$ is induced by the inverse transformation on the smooth elliptic fibers. From the point of view of the modular group, that symmetry is given by minus the identity $\left(-I_{2}\right)$ and corresponds to the perturbative string theory operator $(-)^{F_{\mathrm{L}}} \Omega$, where $\Omega$ is the worldsheet parity and $F_{\mathrm{L}}$ is the left-moving sector spacetime fermion number on the worldsheet. On the hexagram, the map $\mathscr{E}_{i j} \leftrightarrow \mathscr{E}_{j i}$ corresponds to the central involution. It enlarges $\mathscr{S}_{3} \simeq \operatorname{Dih}_{3}$ to $\operatorname{Dih}_{6}$.

\subsection{Some comments on the exotic fibers}

In this section we will comment on the properties of the resolution we have obtained: the enhancement of fibers without increase of the rank, the orientifold picture and a no-go theorem using Batyrev theorem on the birational invariance of Betti numbers in the category of projective algebraic varieties.

(1) Strong coupling: At the points $\Pi_{4}$, the $j$-invariant vanishes. It follows that the string coupling $g_{\mathrm{s}}=(\operatorname{Im} \tau)^{-1}$ is strongly coupled. Interestingly, the string coupling constant is small $(j=\infty)$ anywhere else in the $\mathrm{SU}(5)$ divisor.

\footnotetext{
${ }^{9}$ The projective $E_{6}$ (resp. affine $\tilde{E}_{6}$ ) is composed of six (resp. seven) rational curves. Once the rational curve $C_{0}$ is removed, the remaining curves admit a dual graph of type projective $D_{5}$ (resp. projective $E_{6}$ ) Dynkin diagram.
} 


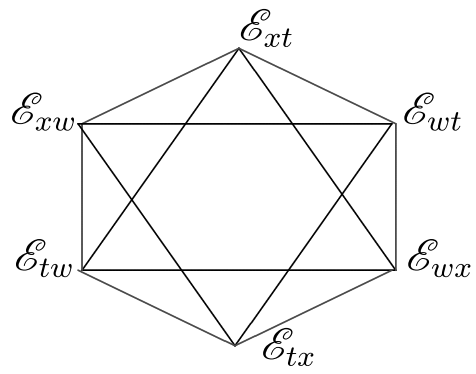

Figure 17: The six small and flat resolutions of the SU(5) variety are related to each other by a network of flop transitions. Each triangles is an orbit of an element of $\mathscr{S}_{3}$ of order 3 . These two orbits are exchanged by any transposition of $\mathscr{S}_{3}$. The group $\mathscr{S}_{3}$ is isomorphic to the dihedral group $\mathrm{Dih}_{3}$ of the triangle. The only permutation which preserves the structure of all fibers is $(x t)$. The involution of the smooth fibers generated by the inverse of the group law of the elliptic fiber induces a birational transformation $\iota$ that acts as $\mathscr{E}_{i j} \mapsto \mathscr{E}_{j i}$ on the six resolutions. In string theory, it corresponds to $(-)^{F_{\mathrm{L}}} \Omega$. On the hexagram, it corresponds to the central involution. It enlarges $\mathscr{S}_{3}$ to $\operatorname{Dih}_{6}$.

(2) Conifold singularities in the orientifold picture: In the type IIB orientifold picture, we consider the double cover over the base ramified at $b_{2}=0$ where $b_{2}=a_{1}^{2}+4 a_{2}$. Using the original Weierstrass equation of the four-fold before the blow-ups, we have $X: \xi^{2}=b_{2}$ with $b_{2}=\beta_{5}^{2}+4 \beta_{4} w$ and $\xi$ a section of $\mathscr{L}$ :

$$
X: \xi^{2}=\beta_{5}^{2}+4 w \beta_{4} .
$$

The elliptic four-fold $Y$ is Calabi-Yau when $\mathscr{L}=K_{B}$ and it follows that when $Y$ is Calabi-Yau, the double cover $X$ is also Calabi-Yau. If we rewrite $X$ as follows

$$
X:\left(\beta_{5}+\xi\right)\left(\beta_{5}-\xi\right)-4 \beta_{4} w=0 .
$$

It is clear that the points $\Pi_{4}: \beta_{5}=\beta_{4}=0$ in the divisor $D_{\mathfrak{s u}(5)}: w=0$ correspond to conifold singularities of $X$ and these points are located on the ramification locus (the orientifold). This has been noticed already by Donagi and Wijnholt [24]. Conifold singularities of three-folds are very well understood and in the context of Calabi-Yau three-folds their physical interpretations have been given by Strominger [35]. In the present case, there is a complication since we are in presence of an 
orientifold symmetry. The two small resolutions of the conifolds are

$$
X_{+}\left\{\begin{array} { l } 
{ \lambda ( \beta _ { 5 } + \xi ) - \mu w = 0 } \\
{ \mu ( \beta _ { 5 } - \xi ) - 4 \lambda \beta _ { 4 } = 0 }
\end{array} \quad \stackrel { \xi \rightarrow - \xi } { - - - \rightarrow } \quad X _ { - } \left\{\begin{array}{l}
\lambda\left(\beta_{5}-\xi\right)-\mu w=0, \\
\mu\left(\beta_{5}+\xi\right)-4 \lambda \beta_{4}=0
\end{array}\right.\right.
$$

where $[\lambda: \mu]$ denotes projective coordinates of a $\mathbb{C P}^{1}$. These two small resolutions $X_{ \pm}$are exchanged by the orientifold involution $\xi \mapsto-\xi$. This behavior is parallel to the one we have discussed for the small resolutions of the four-fold: a discrete symmetry of the singular space becomes a birational transformation between two different small resolutions. We could also consider the blow-up of the conifold points where the singular points are replaced by ruled surfaces $\mathbb{F}_{0}=\mathbb{C P}^{1} \times \mathbb{C P}^{1}$ defined by a quadric equation in $\mathbb{C P}^{3}$ :

$$
u_{1} u_{2}-u_{3} u_{4}=0, \quad\left[u_{1}, u_{2}, u_{3}, u_{4}\right] \in \mathbb{C P}^{3} .
$$

Here, the involution preserves the resolved space but exchanges the two rulings of $\mathbb{F}_{0}$ and the quotient is just a projective plane $\left(\mathbb{F}_{0} / \iota=\mathbb{P}^{2}\right)$. It would be interesting to study the link between the exotic fibers in F-theory and the conifold points of type IIB orientifolds further.

(3) Degeneration without an increase of the rank: The fibers above the points $\Pi_{4}$ are examples of non-transverse collisions without an increase of the number of nodes of the fibers. Collisions without increase of the rank of the singularity has been noticed before [31], but in a different context where exotic fibers had higher dimensional components. It was deduced that in the heterotic dual, the codimension-three singularities corresponded to a point-like degeneration of certain bundles. The model of [31] is studied further in appendix $\mathrm{G}$ of [25]. The codimension-three singularity we have obtained here are different from the one studied in [31] because we have a flat fibration (all the fibers have the same dimension). However, we could easily construct a non-flat resolution $Z \rightarrow Y$ by blowing-up the lines $L_{x}, L_{w}$ and $L_{t}$ after resolving the $I_{5}$ singularity.

(4) Batyrev theorem and a no-go theorem: Using p-adic analysis on algebraic varieties, Batyrev proved that birational Calabi-Yau varieties have the same Betti numbers [20]. This implies in our case that all the six small resolutions we have constructed have the same Betti numbers. Any other small resolution will also have the same Betti numbers since it will be birationally equivalent to the resolutions we have constructed here. This would imply the impossibility to obtain a small resolution where the fibers above the points $\Pi_{4}$ have a dual graph of type $\tilde{E}_{6}$. In other words, the conjectured fiber structure of SU(5) models is impossible since it will have a different Euler characteristic than 
the birational equivalent resolution obtained in this paper. The difference of Euler characteristic is due to the difference of fibers above $\Pi_{4}$. Obviously, a no-go theorem is just as strong as its assumptions. Batyrev theorem is very strong since it only requires the two birational copies $Y_{1}$ and $Y_{2}$ to be smooth irreducible projective algebraic varieties related by a birational map that does not change the canonical class [20].

\section{Conclusions and discussions}

We have presented an explicit desingularization of the elliptically fibered four-fold describing the SU(5) GUT geometry in F-theory. In codimensionone in the base, the resolution of the generic singularity generates a fiber of Kodaira type $I_{5}$ over the SU(5) divisor. After resolving the singularity in codimension-one, there are several left-over singularities in codimension-two and codimension-three. These higher codimension singularities are all visible in a unique patch where they can be described elegantly by an affine binomial variety. The singular locus of the binomial variety consists of a bouquet of three lines $L_{w}, L_{t}, L_{x}$ of conifold singularities all intersecting at a common point $p_{3}$ where the singularity worsen. The binomial geometry admits six small resolutions that we describe both torically and algebraically. In each of these small resolutions, the exceptional locus is a $\mathbb{C P}^{1}$ bundle over the bouquet of three lines $L_{w}, L_{t}, L_{x}$. At the origin of the bouquet, the $\mathbb{C P}^{1}$ fiber enhances to two intersecting $\mathbb{C P}^{1} \mathrm{~s}$. We then obtain a full desingularization of the $\mathrm{SU}(5)$ geometry by pulling back the resolution obtained for the binomial variety. We end up with six small resolutions related to each other by flop transitions.

All the six small resolutions have the same fiber structure in codimensionone and codimension-two in the base and also in codimension-three for the fibers above the points $\beta_{5}=\beta_{3}=0$ in the $\mathrm{SU}(5)$ divisor. In codimension-one we have the $I_{5}$ fiber which described the $\mathrm{SU}(5)$ gauge group. In codimensiontwo, we get enhancement to $\tilde{A}_{5}$ and $\tilde{D}_{5}$, respectively, along a curve $P=$ 0 and $\beta_{5}=0$ in the $\mathrm{SU}(5)$ divisor. The dual graph $\tilde{A}_{5}$ corresponds to a Kodaira fiber of type $I_{6}$ while the dual graph $\tilde{D}_{5}$ corresponds to a Kodaira fiber $I_{1}^{*}$. Another interesting aspect of the resolution is that there are no fibers of type $\tilde{A}_{6}$ in codimension-three although above the points $P=R=0$ in the $\mathrm{SU}(5)$ divisor, the discriminant locus has an enhanced singularity.

The biggest surprise occurs in codimension-three for the fibers above the points $\Pi_{4}: \beta_{4}=\beta_{5}=0$ in the base. Above these points, the six small resolutions do not admit the same dual graph as we can see from figure 18 . 


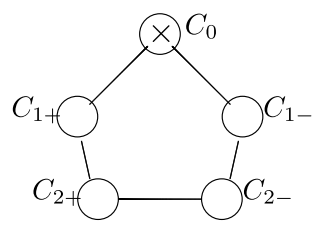

$$
P=0 \quad \searrow \beta_{5}=0
$$
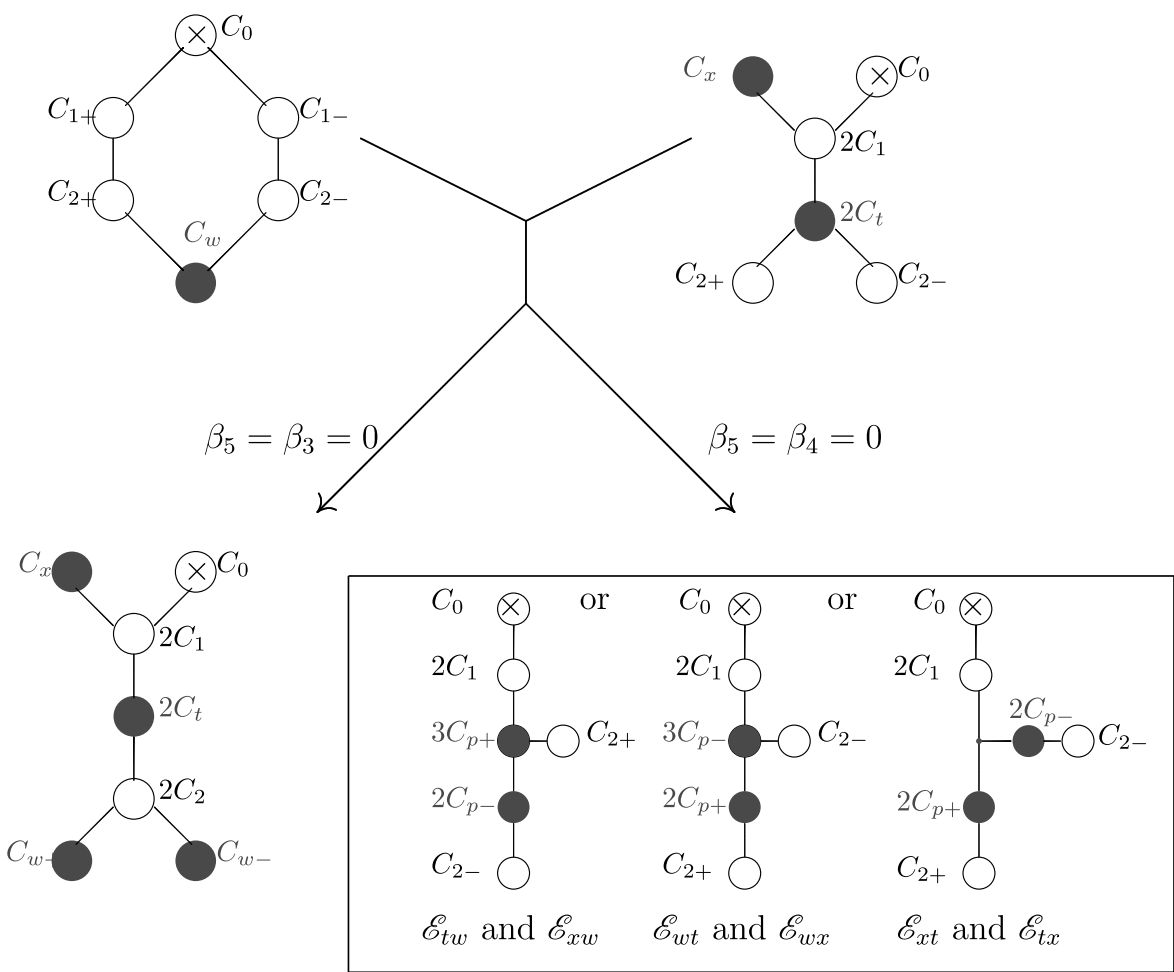

Figure 18: Fiber degeneration of an SU(5) GUTs with a small resolution. Nodes $C_{x}, C_{w}$ and $C_{t}$ are coming from the resolution of the higher codimensional singularities. We have six possible resolutions $\mathscr{E}_{x w}, \mathscr{E}_{w x}, \mathscr{E}_{x t}, \mathscr{E}_{t w}$ and $\mathscr{E}_{w t}$. They are all related to each other by flop transitions related to permutation group of three elements.

More precisely, over the points $\Pi_{4}: \beta_{5}=\beta_{4}=0$ where an affine $\tilde{E}_{6}$ is usually expected in the physics literature, we get a fiber with dual graph $E_{6}$ for four of the six resolutions while the remaining two admits singular fibers whose dual graph is not a Dynkin diagram but a new exotic diagram that we call $\tilde{E}_{6}^{-}=T_{3,3,3}^{-}$: that is a bouquet of three two-chains intersecting at the same point. Such fiber can be seen as a fiber of type $\tilde{E}_{6}$ with the central node contracted to a point. The dual graphs $E_{6}$ and $\tilde{E}_{6}^{-}$have the same 
number of nodes as the graph $\tilde{A}_{5}$ or $\tilde{D}_{5}$. This is an example of enhancement of a singular fiber with conservation of the rank of the fiber. Using Batyrev theorem on the Betti numbers of birational equivalent Calabi-Yau varieties, once can deduce that the conjectured fiber structure of SU(5) models is impossible in the category of projective algebraic varieties. If one specializes the defining equation of the SU(5) models, the type of fibers will change, we will consider such models in a different work.

\section{Acknowledgments}

M.E. would like to thank Murad Alim, Paolo Aluffi, Clay Cordova, Frederik Denef, Ron Donagi, Tamar Fridmann, Antonella Grassi, Jonathan Heckman, Shamit Kachru, Si Li, David Morrison, James $\mathrm{M}^{\mathrm{C}}$ Kernan, Keiji Oguiso, Sakura Schäfer-Nameki, Michael Szydlo, Washington Taylor, Li-Sheng Tseng and Martin Wijnholt for interesting discussions. The content of this paper has been presented in the workshop on Generalized Geometries and String Theory in March 2011 and at the String Math conference in June 2011 and in seminars at Harvard, Stanford, KITP and Caltech. We would like to thank the audience for their comments and suggestions. The authors would like to thank Sheldon Katz for his very useful comments and for pointing out an arithmetic typo in the multiplicity of nodes.

\section{References}

[1] J.C. Baez and J. Huerta, The algebra of grand unified theories, arXiv:0904.1556 [hep-th].

[2] H. Georgi, Lie algebras in particle physics: from isospin to unified theories, Front. Phys. 54 (1982), 1-25.

[3] H. Georgi and S.L. Glashow, Unity of all elementary particle forces, Phys. Rev. Lett. 32 (1974), 438.

[4] T. Friedmann, E. Witten, Unification scale, proton decay, and manifolds of G(2) holonomy, Adv. Theor. Math. Phys. 7 (2003), 577-617; arXiv: hep-th/0211269.

[5] C. Vafa, Evidence for F-theory, Nucl. Phys. B 469 (1996), 403; arXiv:hep-th/9602022.

[6] D.R. Morrison and C. Vafa, Compactifications of F-theory on Calabi-Yau threefolds - I, Nucl. Phys. B 473, 74 (1996); arXiv: hep-th/9602114.

[7] D.R. Morrison and C. Vafa, Compactifications of F-theory on Calabi-Yau threefolds - II, Nucl. Phys. B 476 (1996) 437; arXiv:hep-th/9603161. 
[8] J.H. Schwarz, The power of $M$ theory, Phys. Lett. B 367, 97-103 (1996); hep-th/9510086.

[9] C. Vafa, Geometry of grand unification, arXiv:0911.3008 [math-ph].

[10] A. Sen, Orientifold limit of F-theory Vacua, Phys. Rev. D 55 (1997), 7345; arXiv:hep-th/9702165.

[11] M. Bershadsky, K.A. Intriligator, S. Kachru, D.R. Morrison, V. Sadov and C. Vafa, Geometric singularities and enhanced gauge symmetries, Nucl. Phys. B 481 (1996), 215; arXiv: hep-th/9605200.

[12] S.H. Katz and C. Vafa, Matter from geometry, Nucl. Phys. B 497 (1997), 146; arXiv:hep-th/9606086.

[13] P. Deligne, Courbes elliptiques: formulaire d'après J. Tate in 'Modular Functions of One Variable', IV (Proceedings Internat. Summer School, Univ. Antwerp, Antwerp, 1972), eds. B.J. Birch and W. Kuyk, Lecture Notes in Math., 476, Springer, Berlin, 1975, 53-73.

[14] K. Kodaira, On compact analytic surfaces II, Ann. Math. 77 (1963), 563-626.

[15] A. Néron, Modèles Minimaux des Variétés Abeliennes sur les Corps Locaux et Globaux, Publ. Math. I.H.E.S. 21 (1964), 361-482.

[16] R. Miranda, Smooth models for elliptic threefolds, in 'The Birational Geometry of Degenerations', eds. R. Friedman, D.R. Morrison, Progress in Math., 29, Birkhauser, Basel, 1983, 85-133.

[17] M. Szydlo, Flat regular models of elliptic schemes, Ph.D thesis, Harvard University, 1999.

[18] J. Tate, Algorithm for determining the type of a singular fiber in an elliptic pencil, in 'Modular Functions of One Variable IV', eds. B.J. Birch and W. Kuyk, Lect. Notes Math., 476, Springer-Verlag, Berlin, 1975, 33-52.

[19] T. Fujita, Zariski decomposition and canonical rings of elliptic threefolds, J. Math. Soc. Japan 38 (1986), 19-37.

[20] V.V. Batyrev, Birational Calabi-Yau n-folds have equal Betti numbers, in 'New Trends in Algebraic Geometry (Warwick, 1996)', London Math. Soc., Lecture Notee Ser., 264, Cambridge University Press, Cambridge, 1999, 1-11, alg-geom/9710020

[21] A. Grassi, D.R. Morrison, Group representations and the Euler characteristic of elliptically numbered Calabi-Yau threefolds, J. Algebraic Geom. 12 (2003), 321-356; arXiv:0005196 [math-ag].

[22] B. Andreas and G. Curio, From local to global in F-theory model building, J. Geom. Phys. 60 (2010), 1089; arXiv:0902.4143 [hep-th].

[23] R. Donagi and M. Wijnholt, Model building with F-theory, arXiv:0802.2969 [hep-th]. 
[24] R. Donagi and M. Wijnholt, Higgs bundles and UV completion in Ftheory, arXiv:0904.1218 [hep-th].

[25] C. Beasley, J.J. Heckman and C. Vafa, GUTs and exceptional branes in F-theory - I, J. High Energy Phys. 01 (2009), 058; arXiv:0802. 3391 [hep-th].

[26] A. Collinucci, F. Denef and M. Esole, D-Brane deconstructions in IIB orientifolds, J. High Energy Phys. 02 (2009), 005; arXiv:0805.1573 [hep-th].

[27] P. Aluffi and M. Esole, Chern class identities from tadpole matching in type IIB and F-theory, J. High Energy Phys. 03 (2009), 032; arXiv:0710.2544 [hep-th].

[28] P. Aluffi and M. Esole, New orientifold weak coupling limits in F-theory, J. High Energy Phys. 02 (2010), 020; arXiv:0908.1572 [hep-th].

[29] H. Hayashi, T. Kawano, R. Tatar and T. Watari, Codimension-3 singularities and Yukawa couplings in F-theory, Nucl. Phys. B 823 (2009), 47; arXiv:0901.4941 [hep-th].

[30] S. Cecotti, C. Cordova, J.J. Heckman and C. Vafa, T-Branes and monodromy, arXiv:1010.5780 [hep-th].

[31] P. Candelas, D.E. Diaconescu, B. Florea, D.R. Morrison and G. Rajesh, Codimension-three bundle singularities in F-theory, J. High Energy Phys. 06 (2002), 014; arXiv: hep-th/0009228.

[32] M. Bershadsky, A. Johansen, Colliding singularities in $F$ theory and phase transitions, Nucl. Phys. B 489 (1997), 122-138; arXiv:hep-th/9610111.

[33] B. Teissier, Monomials ideals, binomial ideals, polynomial ideas, in 'Trends in Commutative Algebra', Math. Sci. Res. Inst. Puubl., 51, Cambridge University Press, Cambridge, 2004, 211-246.

[34] M.F. Atiyah, On analytic surfaces with double points, Proc. Roy. Soc. London A, Math. Phys. Eng. Sci. 247 (1958), 237-244.

[35] A. Strominger, Massless black holes and conifolds in string theory, Nucl. Phys. B 451 (1995), 96-108; arXiV:hep-th/9504090.

[36] D.R. Morrison, W. Taylor, Matter and singularities, arXiv:1106.3563 [hep-th].

[37] S. Katz, D.R. Morrison, S. Schafer-Nameki and J. Sully, Tate's algorithm and F-theory, arXiv:1106.3854 [hep-th].

[38] T.W. Grimm, S. Krause and T. Weigand, F-theory GUT Vacua on compact Calabi-Yau fourfolds, J. High Energy Phys. 07 (2010), 037; arXiv:0912.3524 [hep-th]. 
[39] R. Blumenhagen, T.W. Grimm, B. Jurke and T. Weigand, Global F-theory GUTs, Nucl. Phys. B 829 (2010), 325; arXiv:0908.1784 [hep-th].

[40] T.W. Grimm and T. Weigand, On Abelian gauge symmetries and proton decay in global F-theory GUTs, Phys. Rev. D 82 (2010), 086009; arXiv: 1006.0226 [hep-th]. 
